\title{
Sciendo
}

Int. J. of Applied Mechanics and Engineering, 2021, vol.26, No.2, pp.84-106

DOI: 10.2478/ijame-2021-0021

\section{EFFECT OF HALL CURRENTS ON UNSTEADY MAGNETOHYDRODYNAMIC TWO-IONIZED FLUID FLOW AND HEAT TRANSFER IN A CHANNEL}

\author{
T. LINGA RAJU* \\ Department of Engineering Mathematics, $\operatorname{AUCE}(\mathrm{A})$, \\ ANDHRA UNIVERSITY, Visakhapatnam, Pin code: 530003, INDIA \\ E-mail: prof.tlraju@andhrauniversity.edu.in \\ V.GOWRISANKARA RAO \\ Dept. of Mathematics, St. Joseph's College for Women (A), Visakhapatnam, A.P., INDIA
}

\begin{abstract}
An unsteady magnetohydrodynamic (MHD) heat transfer two-fluid flow of ionized gases through a horizontal channel between parallel non-conducting plates, by taking Hall currents into account is studied. The governing partial differential equations that describe the flow and heat transfer under the adopted conditions are solved for the velocity and temperature distributions by a regular perturbation technique. Profiles for the velocity and temperature distributions as well the rates of heat transfer coefficient are presented graphically, and a parametric study is performed. The results reveal that the combined effects of the Hartmann number, Hall parameter, and the ratios of viscosities, heights, electrical and thermal conductivities have a significant impact on an unsteady MHD heat transfer two-ionized fluid flow characteristics.
\end{abstract}

Key words: Hall currents, heat transfer, magnetohydrodynamics (MHD), multi-fluid model, plasma flows.

\section{Introduction}

The progress in contemporary technology has stimulated interest in magnetohydrodynamic (MHD) multi-fluid/or immiscible fluid flow studies due to their widespread application in various specialized fields. For example, the development of a conceptual design for fusion reactors, liquid metal magnetohydrodynamic (MHD) power generators, MHD accelerators, electromagnetic pumps, etc. demands a precise knowledge of heat transfer and thermo-hydraulic mechanisms of two-phase/or two-fluid flow models under the influence of an applied magnetic field. It is also understood that the two-fluid flow provides significant enhancement in heat transfer at certain conditions. Transportation and extraction of the products of oil in geothermal regions are also other obvious applications using a two-phase/or two-fluid flow model to obtain better flow rates in an electromagnetic pump with the added advantage of reducing the power required to pump oil in a pipeline by a suitable addition of water [1]. It is also a known fact that the fluid flows whether Newtonian or non-Newtonian fluid, in general are unsteady/or transient in nature. In particular, a significant number of practical problems that arise while dealing with immiscible fluids are due to of this unsteady nature. Most of the problems related to the petroleum industry, geophysics, plasma physics, magneto-fluid dynamics and so forth, involve multifluid flow situations. It is also beneficial in several practical problems to consider both immiscible fluids as electrically conducting, one of which is a highly electric conducting fluid compared to the other. The fluid of low electrical conductivity compared to the other is useful to minimize the power consumption while pumping the fluid in MHD pumps and flow meters, etc.

In numerous engineering, technological and industrial problems, it is useful to know the amount of heat flow and also the pattern of temperature distribution which the heat transfer creates under an unsteady

\footnotetext{
* To whom correspondence should be addressed
} 
motion in different geometries. Likewise, the oscillatory flows are often utilized in order to augment the heat transfer rates in various "industrial processes". Apart from these, Cowling [2] stated that when the working fluid is an ionized gas where the density is low or the magnetic field is very strong, the effect of Hall currents becomes significant and one cannot ignore the resulting Hall Effect. Therefore, it is of significant concern to study how the outcomes of unsteady MHD two-fluid flow get modified by the presence of Hall currents. The Hall currents are of incredible importance in many astrophysical and geophysical problems, flows of plasma in the MHD power generator, plasma jets, Hall accelerators, and so forth.

Numerous model studies on the effect of Hall currents in the magnetohydrodynamic (MHD) problems of one fluid flow system can be found in literature [3-16]. A great number of studies are also available on MHD two-phase/two-fluid flow problems due to their abundant applications in various domains [17-33]. In most of these studies, the flows are assumed under the steady-state conditions. But in practice, there is a great need to understand the effect of an external body force on the motion of electrically conducting fluids in twodimensional time-reliant circumstances. In spite of the above mentioned studies, we find some investigations that are reported in the literature on the MHD two-fluid flow models with and without Hall effect. To mention a few, Kalra et al. [34] investigated the effect of Hall current and resistivity on the stability of a gas-liquid system. Hyun and Kennel [35] discussed the small amplitude waves in a hot relativistic two-fluid plasma. Sharma and Sharma [36] investigated the MHD two-fluid flow and heat transfer through a horizontal channel. Joseph et al. [37] studied an unsteady MHD free convective two immiscible fluid flow in a horizontal channel with heat and mass transfer. Sivakamini and Govindarajan [38] studied an unsteady MHD flow of two immiscible fluids under chemical reaction in a horizontal channel. Gireesha et al. [39] studied the Hall effects on dusty nanofluid two-phase transient flow past a stretching sheet using KVL model. Raju [40] investigated the MHD heat transfer in two-ionized fluid flow between parallel plates with Hall currents.

All the above-mentioned studies were carried out mostly under the steady-state conditions. And to the best of the author's knowledge, no study has been conducted to investigate the problems in unsteady MHD two-fluid flow and heat transfer of ionized gases through a horizontal channel under the action of an applied transverse magnetic field with the effects of Hall currents. In this paper, we investigate the Hall effect on an unsteady MHD two-fluid flow and heat transfer of ionized gases in a horizontal channel bound by two parallel, non-conducting plates, under the influence of an applied transverse magnetic field. It is expected that this kind of theoretical investigation may have some practical application in numerous fields of engineering disciplines, including chemical, petroleum and in particular, geothermal systems and plasma jets, in the design of MHD power generators, pumps and flow meters, Hall accelerators, and in the cooling process of nuclear reactors and so forth.

\section{Formulation and mathematical analysis of the problem}

The MHD two-ionized fluid flow model under consideration consists of a horizontal channel bounded by two parallel infinite plates extending in the $\mathrm{x}$-and $\mathrm{z}$ - directions, choosing the origin midway between the plates of the Cartesian coordinate system (see Fig.1). The $\mathrm{x}$-axis is taken along a hydrodynamic pressure gradient in the plane parallel to the channel plates but not towards the flow. The channel width is assumed to be extremely large in comparison with the channel height. The two bounding plates of the channel comprise non-conducting (insulating) material and are maintained at a constant temperature $T_{w 1}$ and $T_{w 2}$, where $T_{w 1}$ is the temperature of the boundary at $y=h_{1}$ and that $T_{w 2}$ at $y=h_{2}$. The upper and lower regions of the two fluids are designated as region-I and region-II. They are taken in the regions $0 \leq y \leq h_{1}$ and $-h_{2} \leq y \leq 0$ respectively. We consider a two-dimensional magnetohydrodynamic (MHD) two-fluid flow of ionized gases driven by a common constant pressure gradient $-\frac{\partial p}{\partial x}$ through the horizontal channel between two parallel plates under the transverse electric and magnetic fields. It is supposed that the two-fluid flow is unsteady, laminar and fully developed. Fluids in the region-I and region-II are considered incompressible, immiscible, electrically conducting with different fluid characteristics. Specifically, with different densities $\rho_{l}, \rho_{2}$, 
viscosities $\mu_{1}, \mu_{2}$, electrical conductivities $\sigma_{01}, \sigma_{02}$ and thermal conductivities $\mathrm{K}_{1}, \mathrm{~K}_{2}$. A magnetic field of constant strength $B_{0}$ is applied in the y-direction i.e., normal to the channel plates, and the effects of Hall currents are also taken into account. The induced magnetic field is disregarded by considering that the magnetic Reynolds number is small. The two boundaries of the channel are rigid and the interface between two immiscible fluids is flat, stress-free and undisturbed. It is taken for granted that the thermal boundary conditions are valid all over on the infinite channel plates. The thermal conduction in the flow direction and electron heating are ignored. All physical quantities except pressure in this study are the functions of ' $y$ ' and ' $t$ ' only as the plates are infinite in extent along the $\mathrm{x}$ - and z-directions. With these assumptions and in view of existing studies in the literature, the governing equations are formulated based on the fundamental equations for an unsteady MHD flow of neutral fully ionized gas in the presence of Hall currents. Accordingly, the equations for an unsteady MHD two-ionized fluid flow and heat transfer in a channel of parallel nonconducting plates under the action of transverse electric and magnetic fields in two-fluid regions are specified.

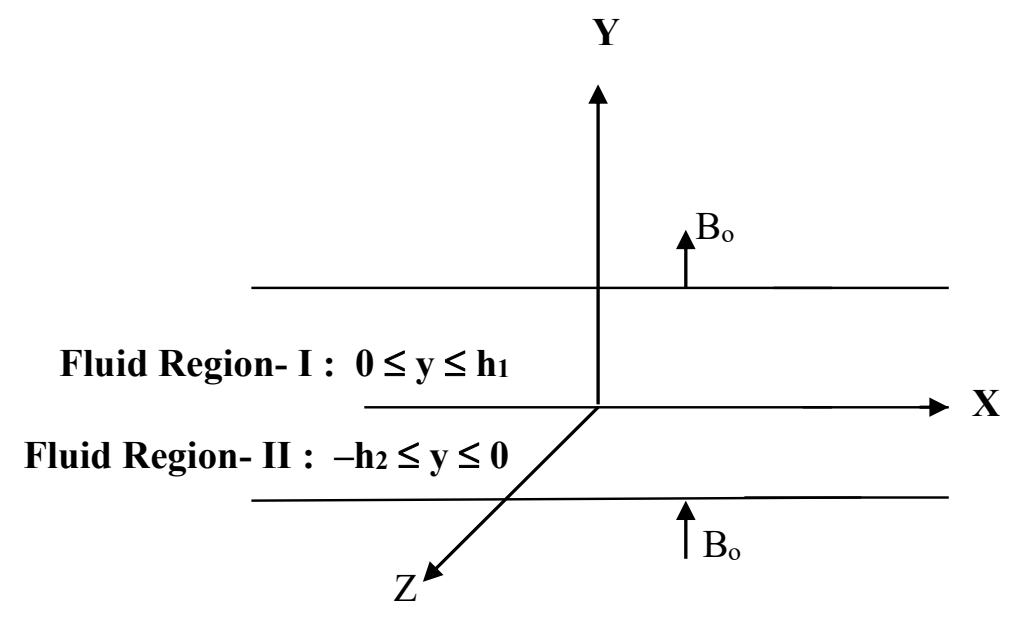

Fig.1. The two-fluid flow model and co-ordinate system.

The governing equations in this investigation are the equations of motion, current and energy along with the boundary, and interface conditions. Under the above-stated suppositions and adopting the analysis as in the investigations of Sato [5] and Raju [40], we assume in the fundamental equations that the fluid velocity as $\bar{V}_{i}=\left(u_{i}, 0, w_{i}\right)$, the magnetic flux intensity as $\overline{\mathrm{B}}=\left(0, B_{0}, 0\right)$, the current density as $\overline{\mathrm{J}_{i}}=\left(J_{i x}, 0, J_{i z}\right)$, the electric field as $\overline{E_{i}}=\left(\mathrm{E}_{i x}, 0, E_{i z}\right)$ and $J_{i}^{2}=J_{i x}^{2}+J_{i z}^{2},(i=1,2)$ for both fluid regions. Hence, the governing equations in the two-fluid regions (that is, for the fluids in the upper and lower regions namely, Region-I and Region-II) are simplified and obtained as:

\section{Region-I:}

$$
\begin{aligned}
& \rho_{1} \frac{\partial u_{1}}{\partial t}=\mu_{1} \frac{\partial^{2} u_{1}}{\partial y^{2}}-\left\{1-s\left(1-\frac{\sigma_{11}}{\sigma_{01}}\right)\right\} \frac{\partial p}{\partial x}+\left\{-\sigma_{11}\left(E_{1 z}+u_{1} B_{0}\right)+\sigma_{21}\left(E_{1 x}-w_{1} B_{0}\right)\right\} B_{0}, \\
& \rho_{1} \frac{\partial w_{1}}{\partial t}=\mu_{1} \frac{\partial^{2} w_{1}}{\partial y^{2}}+s \frac{\partial p}{\partial x} \frac{\sigma_{21}}{\sigma_{01}}+\left\{\sigma_{11}\left(E_{1 x}-w_{1} B_{0}\right)+\sigma_{21}\left(E_{1 z}+u_{1} B_{0}\right)\right\} B_{0},
\end{aligned}
$$




$$
\begin{aligned}
& \rho_{1} c_{p_{1}} \frac{\partial T_{1}}{\partial t}-K_{1} \frac{\partial^{2} T_{1}}{\partial y^{2}}=\mu_{1}\left[\left(\frac{\partial u_{1}}{\partial y}\right)^{2}+\left(\frac{\partial w_{1}}{\partial y}\right)^{2}\right]+\frac{J_{1}^{2}}{\sigma_{01}} \\
& J_{1 x}=\sigma_{11} E_{1 x}-B_{0} \sigma_{11} w_{1}+\sigma_{21} E_{1 z}+B_{0} \sigma_{21} u_{1}+\frac{\sigma_{21}}{\sigma_{01}} \frac{s}{B_{0}} \frac{\partial p}{\partial x} \\
& J_{1 z}=\left(\frac{E_{1 z}}{B_{0}}+u_{1}\right) \sigma_{11}-\left(\frac{E_{1 x}}{B_{0}}-w_{1}\right) \sigma_{21}+\left(\frac{\sigma_{11}}{\sigma_{01}}-1\right) \frac{s}{B_{0}} \frac{\partial p}{\partial x}
\end{aligned}
$$

\section{Region-II:}

$$
\begin{aligned}
& \rho_{2} \frac{\partial u_{2}}{\partial t}=\mu_{2} \frac{\partial^{2} u_{2}}{\partial y^{2}}-\left\{1-s \frac{\partial p}{\partial x}\left(1-\frac{\sigma_{12}}{\sigma_{02}}\right)\right\}+\left\{-\sigma_{12}\left(E_{2 z}+u_{2} B_{0}\right)+\sigma_{22}\left(E_{2 x}-w_{2} B_{0}\right)\right\} B_{0}, \\
& \rho_{2} \frac{\partial w_{2}}{\partial t}=\mu_{2} \frac{\partial^{2} w_{2}}{\partial y^{2}}+\left(s \frac{\partial p}{\partial x} \frac{\sigma_{22}}{\sigma_{02}}\right)+\left\{\sigma_{12}\left(E_{2 x}-w_{2} B_{0}\right)+\sigma_{22}\left(E_{2 z}+u_{2} B_{0}\right)\right\} B_{0}, \\
& \rho_{2} c_{p_{2}} \frac{\partial T_{2}}{\partial t}-K_{2} \frac{\partial^{2} T_{2}}{\partial y^{2}}=\mu_{2}\left\{\left(\frac{\partial u_{2}}{\partial y}\right)^{2}+\left(\frac{\partial w_{2}}{\partial y}\right)^{2}\right\}+\frac{J_{2}^{2}}{\sigma_{02}} \\
& J_{2 x}=\sigma_{12} E_{2 x}-B_{0} \sigma_{12} w_{2}+\sigma_{22} E_{2 z}+B_{0} \sigma_{22} u_{2}+\frac{\sigma_{22}}{\sigma_{02}} \frac{s}{B_{0}} \frac{\partial p}{\partial x} \\
& J_{2 z}=\left(\frac{E_{2 z}}{B_{0}}+u_{2}\right) \sigma_{12}-\left(\frac{E_{2 x}}{B_{0}}-w_{2}\right) \sigma_{22}+\left(\frac{\sigma_{12}}{\sigma_{02}}-1\right) \frac{s}{B_{0}} \frac{\partial p}{\partial x}
\end{aligned}
$$

where the subscripts 1 and 2 in the above equations refer to the quantities for region-I and II respectively. The quantities $\mathrm{u}_{1}, \mathrm{u}_{2}$ and $\mathrm{w}_{1}, \mathrm{w}_{2}$, are the velocity components along the $\mathrm{x}$-and $\mathrm{z}$ - directions in the two-fluid regions respectively. These distributions are known as primary and secondary velocity distributions. The notations $E_{i x}$ and $E_{i z}, J_{i x}$ and $J_{i z}(i=1,2)$ are the $x$-and $z$-components of the electric field, and the current densities respectively. The quantities $T_{1}, T_{2}$ are the temperatures, $c_{p i}(i=1,2)$ is the specific heat at constant pressure. The quantity, $s=p_{e} / p$ is the proportion of electron pressure to the total pressure, where the values of $s=\frac{1}{2}$ and about zero correspond to the neutral fully-ionized plasma and weakly-ionized gas, respectively. The quantities $\sigma_{11}, \sigma_{12}$ and $\sigma_{21}, \sigma_{22}$ are the modified conductivities parallel and normal to the direction of the electric field, respectively. Since $T_{w 1}$ and $T_{w 2}$ being constant everywhere on the upper and lower plates, respectively, so $\frac{\partial T_{1}}{\partial x}=0, \frac{\partial T_{1}}{\partial z}=0, \frac{\partial T_{2}}{\partial x}=0, \frac{\partial T_{2}}{\partial z}=0$ and $T_{1}, T_{2}$ are finite everywhere in the two-fluid regions, and these are functions of ' $y$ ' and ' $t$ ' only. The fluid velocity and shear stress are assumed to be continuous over the interface $y=0$. The conditions on the temperature field force the isothermal boundary conditions at the two plates, also the continuity of temperature and heat flux at the interface $y=0$. 
The boundary and interface conditions on velocity distributions are

$$
\begin{array}{ll}
u_{l}\left(h_{1}\right) \quad \text { and } \quad w_{1}\left(h_{1}\right)=0 & \text { for } t \leq 0, \\
u_{1}\left(h_{1}\right) \quad \text { and } \quad w_{l}\left(h_{1}\right)=\text { Real } \quad \text { of } \quad \varepsilon e^{i \omega t} \quad \text { for } t>0, & \\
u_{2}\left(-h_{2}\right)=0, \quad w_{2}\left(-h_{2}\right)=0, & w_{l}(0)=w_{2}(0) \quad \text { for } \quad h_{1}=h_{2} . \\
u_{1}(0)=u_{2}(0), & \quad \text { and } \quad \mu_{1} \frac{\partial w_{1}}{\partial y}=\mu_{2} \frac{\partial w_{2}}{\partial y} \quad \text { at } \quad y=0 .
\end{array}
$$

The isothermal boundary and interface conditions for temperature for both fluids are

$$
\begin{aligned}
& T_{1}\left(h_{1}\right)=T_{w 1}, \quad T_{2}\left(-h_{2}\right)=T_{w 2}, \quad T_{1}(0)=T_{2}(0) \quad \text { for } \quad h_{1}=h_{2}, \\
& K_{1} \frac{\partial T_{1}}{\partial y}=K_{2} \frac{\partial T_{2}}{\partial y} \quad \text { at } \quad y=0 .
\end{aligned}
$$

The following non-dimensional variables are used to formulate the governing equations $(2.1)$ to $(2.10)$ and (2.11) to (2.15) as dimensionless:

$$
\begin{aligned}
& u_{1}^{\bullet}=\frac{u_{1}}{u_{p}}, \quad u_{2}^{\bullet}=\frac{u_{2}}{u_{p}}, \quad w_{1}^{\bullet}=\frac{w_{1}}{u_{p}}, \quad w^{\bullet}{ }_{2}=\frac{w_{2}}{u_{p}}, \quad y_{i}^{\bullet}=\frac{y_{i}}{h_{i}}, \\
& u_{p}=-\frac{\partial p}{\partial x} \frac{h_{1}^{2}}{\mu_{1}}, \quad t^{\bullet}=\frac{\mu_{i} t}{\rho_{i} h_{i}^{2}}, \quad \omega^{\bullet}=\frac{\omega h_{i}^{2} \rho_{i}}{\mu_{i}}, \quad(i=1,2), \quad m_{i x}=\frac{E_{i x}}{B_{0} u_{p}}, \\
& m_{i z}=\frac{E_{i z}}{B_{0} u_{p}}, \quad I_{i x}=\frac{J_{i x}}{\sigma_{0 i} B_{0} u_{p}}, \quad I_{i z}=\frac{J_{i z}}{\sigma_{0 i} B_{0} u_{p}}, \quad J_{i}^{2}=J_{i x}^{2}+J_{i z}^{2}, \\
& M^{2}=B_{0}{ }_{0} h^{2}{ }_{1}\left(\frac{\sigma_{01}}{\mu_{1}}\right), \quad \alpha=\frac{\mu_{1}}{\mu_{2}}, h=\frac{h_{2}}{h_{1}}, \quad \rho=\frac{\rho_{2}}{\rho_{1}}, \quad \sigma_{0}=\frac{\sigma_{01}}{\sigma_{02}}, \\
& \left.\sigma_{01}=\frac{\sigma_{12}}{\sigma_{11}}, \quad \sigma_{02}=\frac{\sigma_{22}}{\sigma_{21}}, \quad \frac{\sigma_{11}}{\sigma_{01}}=\frac{1}{1+m^{2}}, \quad \frac{\sigma_{21}}{\sigma_{01}}=\frac{m}{1+m^{2}}, \quad m=\frac{\omega_{e}}{\tau}+\frac{1}{\tau_{e}}\right) \\
& \beta=\frac{K_{1}}{K_{2}}, \quad \theta_{i}=\frac{T_{i}-T_{w i}}{\left(u_{p}{ }^{2} \mu_{1} / K_{i}\right)}
\end{aligned}
$$


where $M^{2}$ is Hartmann number, $\alpha$ is ratio of viscosities, $\mathrm{h}$ is ratio of the heights, $\rho$ is ratio of densities, $\sigma_{0}$ is ratio of electrical conductivities, $m$ is Hall parameter, $\beta$ is ratio of thermal conductivities of the two fluids, $\theta_{i}$ is temperature distribution, $\omega_{e}$ is the gyration frequency of electrons; $\tau$ and $\tau_{\mathrm{e}}$ are the mean collision time between electron and ion, electron and neutral particles individually. The above expression for the Hall parameter ' $m$ ' which is valid on account of partially-ionized gases agrees with that of fully-ionized gases when $\tau_{\mathrm{e}}$ approaches infinity.

By making use of the above transformations (2.16) and simply ignoring the asterisks, the non-dimensional forms of equations (2.1) to (2.10) become:

\section{Region-I}

$$
\begin{aligned}
& \frac{\partial u_{1}}{\partial t}=\frac{\partial^{2} u_{1}}{\partial y^{2}}-\frac{M^{2}}{1+m^{2}}\left(m_{1 z}+u_{1}\right)+\frac{m M^{2}}{1+m^{2}}\left(m_{1 x}-w_{1}\right)+\beta_{1}, \\
& \frac{\partial w_{1}}{\partial t}=\frac{\partial^{2} w_{1}}{\partial y^{2}}+\frac{M^{2}}{1+m^{2}}\left(m_{1 x}-w_{1}\right)+\frac{m M^{2}}{1+m^{2}}\left(m_{1 z}+u_{1}\right)+\beta_{2}, \\
& \frac{\partial \theta_{1}}{\partial t}=\frac{1}{P_{r}} \frac{\partial^{2} \theta_{1}}{\partial y^{2}}+\left\{\left(\frac{\partial \mathrm{u}_{1}}{\partial \mathrm{y}}\right)^{2}+\left(\frac{\partial w_{1}}{\partial \mathrm{y}}\right)^{2}\right\}+M^{2} I_{1}^{2}, \\
& I_{1 x}=\frac{1}{1+m^{2}}\left(m_{1 x}-w_{1}\right)+\frac{m}{1+m^{2}}\left(m_{l z}+u_{1}\right)-\frac{s m}{\left(1+m^{2}\right) M^{2}}, \\
& I_{1 z}=\frac{1}{1+m^{2}}\left(m_{l z}+u_{1}\right)-\frac{m}{1+m^{2}}\left(m_{1 x}-w_{l}\right)+\frac{s}{M^{2}}\left(1-\frac{m}{1+m^{2}}\right), \\
& I_{1}{ }^{2}=I_{1 x}{ }^{2}+I_{l z}{ }^{2} .
\end{aligned}
$$

\section{Region-II}

$$
\begin{aligned}
& \frac{\partial u_{2}}{\partial t}=\frac{\partial^{2} u_{2}}{\partial y^{2}}-\frac{\alpha \sigma_{1} h^{2} M^{2}}{1+m^{2}}\left(m_{2 z}+u_{2}\right)+\frac{m h^{2} M^{2} \alpha \sigma_{2}}{1+m^{2}}\left(m_{2 x}-w_{2}\right)+\alpha h^{2} \beta_{3}, \\
& \frac{\partial w_{2}}{\partial t}=\frac{\partial^{2} w_{2}}{\partial y^{2}}+\frac{\alpha \sigma_{1} h^{2} M^{2}}{1+m^{2}}\left(m_{2 x}-w_{2}\right)+\frac{m h^{2} M^{2} \alpha \sigma_{2}}{1+m^{2}}\left(m_{2 z}+u_{2}\right)+\alpha h^{2} \beta_{4}, \\
& \frac{\partial \theta_{2}}{\partial t}=\frac{1}{P_{r}} \frac{\partial^{2} \theta_{2}}{\partial y^{2}}+\frac{\beta}{\alpha}\left\{\left(\frac{\partial u_{2}}{\partial y}\right)^{2}+\left(\frac{\partial w_{2}}{\partial y}\right)^{2}\right\}+\sigma \beta h^{2} M^{2} I_{2}{ }^{2},
\end{aligned}
$$




$$
\begin{aligned}
& I_{2 x}=\frac{\sigma_{0} \sigma_{01}}{1+m^{2}}\left(m_{2 x}-w_{2}\right)+\frac{m \sigma_{0} \sigma_{02}}{1+m^{2}}\left(m_{2 z}+u_{2}\right)-\frac{s m \sigma_{0}{ }^{2} \sigma_{02}}{\left(1+m^{2}\right) M^{2}}, \\
& I_{2 z}=\frac{\sigma_{0} \sigma_{01}}{1+m^{2}}\left(m_{2 z}+u_{2}\right)-\frac{m \sigma_{0} \sigma_{02}}{1+m^{2}}\left(m_{2 x}-w_{2}\right)+\left(1-\frac{\sigma_{0} \sigma_{01}}{1+m^{2}}\right) \frac{s \sigma_{0}}{M^{2}}, \\
& I_{2}{ }^{2}=I_{2 x}{ }^{2}+I_{2 z}{ }^{2}
\end{aligned}
$$

where,

$$
k_{1}=1-s\left(\frac{1}{1+m^{2}}\right), \quad k_{2}=\frac{-s m}{1+m^{2}}, k_{3}=1-s\left(1-\frac{\sigma_{0} \sigma_{01}}{1+m^{2}}\right), \quad k_{4}=\frac{-s \sigma_{0} \sigma_{02} m}{1+m^{2}}
$$

The boundary and interface conditions on $u_{1}, w_{1}$ and $u_{2}, w_{2}$ are given by

$$
\begin{aligned}
& u_{1}(1)=0 \quad \text { for } \quad t \leq 0, \quad w_{1}(1)=0 \quad \text { and } \quad \text { for } t \leq 0, \\
& u_{1}(1)=\varepsilon \cos \omega t \quad \text { for } \quad t>0 \quad w_{1}(1)=\varepsilon \cos \omega t \quad \text { for } t>0, \\
& u_{2}(-1)=0, \quad w_{2}(-1)=0, \\
& u_{1}(0)=u_{2}(0), \quad w_{1}(0)=w_{2}(0), \\
& \frac{\partial u_{1}}{\partial y}=(1 / \alpha h) \frac{\partial u_{2}}{\partial y}, \quad \frac{\partial w_{1}}{\partial y}=(1 / \alpha h) \frac{\partial w_{2}}{\partial y} \quad \text { and } \quad \mu_{1} \frac{\partial w_{1}}{\partial y}=\mu_{2} \frac{\partial w_{2}}{\partial y} \quad \text { at } \quad y=0
\end{aligned}
$$

The boundary and interface conditions on temperature for both fluids are

$$
\theta_{1}(1)=0, \quad \theta_{2}(-1)=0, \quad \theta_{1}(0)=\theta_{2}(0) \quad \text { and } \quad \frac{\partial \theta_{1}}{\partial y}=\frac{1}{\beta h} \frac{\partial \theta_{2}}{\partial y} \quad \text { at } \quad y=0
$$

\section{Solutions to the problem}

The governing momentum and energy equations (2.17)-(2.19) and (2.22)-(2.24) are to be solved under the boundary and interface conditions (2.27)-(2.31) for the velocity and temperature distributions in both fluid regions. In reality, these equations are coupled partial differential equations that cannot be solved in closed form. Anyway, they can be reduced to ordinary linear differential equations by assuming the following twoterm series (Regular perturbation method of first order):

$$
\begin{aligned}
& u_{l}(\mathrm{y}, \mathrm{t})=u_{01}(\mathrm{y})+\varepsilon \cos \omega t u_{11}(y) \quad \text { and } \quad w_{l}(y, t)=w_{01}(y)+\varepsilon \cos \omega t w_{11}(y) \text {, } \\
& u_{2}(y, t)=u_{02}(y)+\varepsilon \cos \omega t u_{12}(y) \quad \text { and } \quad w_{2}(y, t)=w_{02}(y)+\varepsilon \cos \omega t w_{12}(y) \text {, } \\
& \theta_{1}(y, t)=\theta_{01}(y)+\varepsilon \cos \omega t \theta_{11}(y) \quad \text { and } \quad \theta_{2}(y, t)=\theta_{02}(y)+\varepsilon \cos \omega t \theta_{12}(y) \text {, }
\end{aligned}
$$


in which the terms $u_{01}(y), u_{02}(y), w_{01}(y), w_{02}(y)$ and $\theta_{01}(y), \theta_{02}(y)$ are the velocity and temperature distributions in the two-fluid regions under the steady part, while $u_{11}(y), u_{12}(y), w_{11}(y), w_{12}(y)$ and $\theta_{11}(y), \theta_{12}(y)$ are the related time dependent components of solution to the problem.

In order to combine Eqs (2.17) and (2.18) into a single equation and for simplicity, we introduce a complex function $q_{1}(y, t)=u_{1}(y, t)+i w_{l}(y, t)$; likewise $q_{2}(y, t)=u_{2}(y, t)+i w_{2}(y, t)$ to combine Eqs $(2.22)$ and (2.23). Thereby utilizing the expressions given in Eqs (3.1)-(3.3) into Eqs (2.17)-(2.19) and (2.22)-(2.24) and then separating the steady and transient time-varying parts, we obtain the differential equations in terms of the complex notations, namely $q_{01}, q_{11}, q_{02}$ and $q_{12}$; where $q_{01}=u_{01}+i w_{01}, q_{11}=u_{11}+i w_{11}$, $q_{02}=u_{02}+i w_{02}, q_{12}=u_{12}+i w_{12}$. The resulting linear differential equations are solved analytically by utilizing the boundary and interface conditions. The closed form solutions for both steady and transient-time dependent parts are obtained separately so as to acquire the final solutions to the unsteady-state problem in two-fluid regions.

The solutions for primary and secondary velocity distributions, namely $u_{1}, u_{2}$ and $w_{1}, w_{2}$ respectively in terms of the complex notations are given by

$$
\begin{aligned}
q_{1}(y, t) & =q_{01}(y)+\varepsilon \cos \omega t \cdot q_{11}(y) \\
& =A_{1} e^{m_{1} y}+A_{2} e^{m_{2} y}-\frac{a_{2}}{a_{1}}+\varepsilon \cos \omega t\left(A_{5} e^{m_{5} y}+A_{6} e^{m_{6} y}\right), \\
q_{2}(y, t) & =q_{02}(y)+\varepsilon \cos \omega t \cdot q_{12}(y) \\
& =A_{3} e^{m_{3} y}+A_{4} e^{m_{4} y}-\frac{a_{4}}{a_{3}}+\varepsilon \cos \omega t\left(A_{7} e^{m_{7} y}+A_{8} e^{m_{8} y}\right)
\end{aligned}
$$

where

$$
u_{l}(y, t)=\frac{q_{1}(y, t)+\overline{q_{1}(y, t)}}{2}, \quad w_{l}(y, t)=\frac{q_{l}(y, t)-\overline{q_{l}(y, t)}}{2 i},
$$

and similarly for $u_{2}(y, t)$ and $w_{2}(y, t)$.

The mean velocity distributions in complex notation are

$$
\begin{aligned}
& q_{m_{1}}=u_{m_{1}}+i w_{m_{1}}=\frac{\left(e^{m_{1}}-1\right) A_{1}}{m_{1}}+\frac{\left(e^{m_{2}}-1\right) A_{2}}{m_{2}}-\frac{a_{2}}{a_{1}}+A_{9}, \\
& q_{m_{2}}=u_{m_{2}}+i w_{m_{2}}=\frac{\left(e^{m_{3}}-1\right) A_{3}}{m_{3}}+\frac{\left(e^{m_{4}}-1\right) A_{4}}{m_{4}}-\frac{a_{4}}{a_{3}}+A_{10}
\end{aligned}
$$

where

$$
u_{m_{1}}=\frac{q_{m_{1}}+\overline{q_{m_{1}}}}{2}, \quad w_{m_{1}}=\frac{q_{m_{1}}-\overline{q_{m_{1}}}}{2 i}, \quad u_{m_{2}}=\frac{q_{m_{2}}+\overline{q_{m_{2}}}}{2}, \quad w_{m_{2}}=\frac{q_{m_{2}}-\overline{q_{m_{2}}}}{2 i} .
$$

Consequently, by utilizing the above expressions (3.4)-(3.6), we solve the corresponding energy equations for both steady and transient time-dependent components separately. The final solutions to the unsteady problem of investigation for the temperature distribution in the two regions and the rate of heat transfer coefficients at the two plates are obtained as: 


\section{Region-I:}

$$
\begin{aligned}
& \theta_{1}(y, t)=\theta_{01}(y)+\varepsilon \cos \omega t \theta_{11}(y)=B_{1} y+B_{2}+b_{18} e^{\left(m_{1}+\overline{m_{1}}\right) y}+b_{19} e^{\left(m_{1}+\overline{m_{2}}\right) y}+ \\
& +b_{20} e^{\left(m_{2}+\overline{m_{1}}\right) y}++b_{21} e^{\left(m_{2}+\overline{m_{2}}\right) y}+b_{22} e^{m_{1} y}+b_{23} e^{m_{2} y}+b_{24} e^{\overline{m_{1}} y}+b_{25} e^{\overline{m_{2}} y}+b_{26} y^{2}+ \\
& +\varepsilon \cos \omega t\left\{B_{5} e^{(-\sqrt{\omega \tan \omega t}) y}+B_{6} e^{(\sqrt{\omega \tan \omega t}) y}+b_{70} e^{\left(m_{5}+\overline{m_{5}}\right) y}+b_{71} e^{\left(m_{5}+\overline{m_{6}}\right) y}+\right. \\
& \left.+b_{72} e^{\left(m_{6}+\overline{m_{5}}\right) y}+b_{73} e^{\left(m_{6}+\overline{m_{6}}\right) y}+b_{74} e^{m_{5} y}+b_{75} e^{m_{6} y}+b_{76} e^{\overline{m_{5}} y}+b_{77} e^{\overline{m_{6}} y}\right\} .
\end{aligned}
$$

The rate of heat transfer coefficient at the upper plate, $N u_{1}=-\left(\frac{\partial \theta_{1}}{\partial y}\right)_{\text {at } y=1}$ is

$$
\begin{aligned}
& \mathrm{Nu}_{1}=-\left\{2 b_{26}+B_{1}+b_{18}\left(m_{1}+\overline{m_{1}}\right) e^{\left(m_{1}+\overline{m_{1}}\right)}+b_{19}\left(m_{1}+\overline{m_{2}}\right) e^{\left(m_{1}+\overline{m_{2}}\right)}+\right. \\
& +b_{20}\left(m_{2}+\overline{m_{1}}\right) e^{\left(m_{2}+\overline{m_{1}}\right)}+b_{21}\left(m_{2}+\overline{m_{2}}\right) e^{\left(m_{2}+\overline{m_{2}}\right)}+b_{22} m_{1} e^{m_{1}}+b_{23} m_{2} e^{m_{2}}+b_{24} \overline{m_{1}} e^{\overline{m_{1}}}+ \\
& +b_{25} \overline{m_{2}} e^{\overline{m_{2}}}+\varepsilon \cos \omega t\left[B_{5}(\sqrt{-\omega \tan \omega t}) e^{(\sqrt{-\omega \tan \omega t})}+B_{6}(\sqrt{\omega \tan \omega t}) e^{(\sqrt{\omega \tan \omega t})}+\right. \\
& +b_{70}\left(m_{5}+\overline{m_{5}}\right) e^{\left(m_{5}+\overline{m_{5}}\right)}+b_{71}\left(m_{5}+\overline{m_{6}}\right) e^{\left(m_{5}+\overline{m_{6}}\right)}+b_{72}\left(m_{6}+\overline{m_{5}}\right) e^{\left(m_{6}+\overline{m_{5}}\right)}+ \\
& \left.\left.+b_{73}\left(m_{6}+\overline{m_{6}}\right) e^{\left.\left(m_{6}+\overline{m_{6}}\right)\right)}+b_{74} m_{5} e^{m_{5}}+b_{75} m_{6} e^{m_{6}}+b_{76} \overline{m_{5}} e^{\overline{m_{5}}}+b_{77} \overline{m_{6}} e^{\overline{m_{6}}}\right]\right\} .
\end{aligned}
$$

\section{Region-II:}

$$
\begin{aligned}
& \theta_{2}(y, t)=\theta_{02}(\mathrm{y})+\varepsilon \cos \omega t \theta_{12}(y)=B_{3} y+B_{4}+b_{44} e^{\left(m_{3}+\overline{m_{3}}\right) y}+b_{45} e^{\left(m_{3}+\overline{m_{4}}\right) y}+ \\
& +b_{46} e^{\left(m_{4}+\overline{m_{3}}\right) y}+b_{47} e^{\left(m_{4}+\overline{m_{4}}\right) y}+b_{48} e^{m_{3} y}+b_{49} e^{m_{4} y}+b_{50} e^{\overline{m_{3}} y}+b_{51} e^{\overline{m_{4}} y}+ \\
& +b_{52} y^{2}+\varepsilon \cos \omega t\left\{B_{7} e^{(\sqrt{-\omega \tan \omega t}) y}+B_{8} e^{(\sqrt{\omega \tan \omega t}) y}+b_{94} e^{\left(m_{7}+\overline{m_{7}}\right) y}+b_{95} e^{\left(m_{7}+\overline{m_{8}}\right) y}\right. \\
& \left.+b_{96} e^{\left(m_{8}+\overline{m_{7}}\right) y}+b_{97} e^{\left(m_{8}+\overline{m_{8}}\right) y}+b_{98} e^{m_{7} y}+b_{99} e^{m_{8} y}+b_{100} e^{\overline{m_{7} y}}+b_{101} e^{\overline{m_{8} y}}\right\} .
\end{aligned}
$$

The rate of heat transfer coefficient at the lower plate, $\mathrm{Nu}_{2}=\left.\frac{1}{\beta h}\left(\frac{\partial \theta_{2}}{\partial y}\right)\right|_{y=-1}$ is 


$$
\begin{aligned}
& \mathrm{Nu}_{2}=\frac{1}{\beta h}\left[B_{3}-2 b_{52}+b_{44}\left(m_{3}+\overline{m_{3}}\right) e^{-\left(m_{3}+\overline{m_{3}}\right)}+b_{45}\left(m_{3}+\overline{m_{4}}\right) e^{-\left(m_{3}+\overline{m_{4}}\right)}+\right. \\
& +b_{46}\left(m_{4}+\overline{m_{3}}\right) e^{-\left(m_{4}+\overline{m_{3}}\right)}+b_{47}\left(m_{4}+\overline{m_{4}}\right) e^{-\left(m_{4}+\overline{m_{4}}\right)}+b_{48} m_{3} e^{-m_{3}}+b_{49} m_{4} e^{-m_{4}}+ \\
& +b_{50} \overline{m_{3}} e^{-\overline{m_{3}}}+b_{51} \overline{m_{4}} e^{-\overline{m_{4}}}+\varepsilon \cos \omega t\left\{B_{7}(\sqrt{-\omega \tan \omega t}) e^{(\sqrt{\omega \tan \omega t})}+\right. \\
& +B_{8}(\sqrt{\omega \tan \omega t}) e^{(\sqrt{\omega \tan \omega t})}+b_{94}\left(m_{7}+\overline{m_{7}}\right) e^{-\left(m_{7}+\overline{m_{7}}\right)}+b_{95}\left(m_{7}+\overline{m_{8}}\right) e^{-\left(m_{7}+\overline{m_{8}}\right)}+ \\
& +b_{96}\left(m_{8}+\overline{m_{7}}\right) e^{-\left(m_{8}+\overline{m_{7}}\right)}+b_{97}\left(m_{8}+\overline{m_{8}}\right) e^{-\left(m_{8}+\overline{m_{8}}\right)}+b_{98} m_{7} e^{-m_{7}}+b_{99} m_{8} e^{-m_{8}}+ \\
& \left.+b_{100} \overline{m_{7}} e^{-\overline{m_{7}}}+b_{101} \overline{m_{8}} e^{-\overline{m_{8}}}\right\} .
\end{aligned}
$$

\section{Results and discussion}

In order to solve the governing partial differential equations, a two-term series (i.e., a regular perturbation method of first order) is adopted for velocity distributions, namely the primary and secondary velocity distributions $u_{1}, u_{2}$ and $w_{1}, w_{2}$, and temperature distributions $\theta_{1}, \theta_{2}$ in the two-regions. Related computational estimation for the different sets of values of the governing parameters is illustrated graphically. Profiles for the velocity and temperature distributions in the two regions as well as the rate of heat transfer coefficients are shown in Figs. 2 to 22.

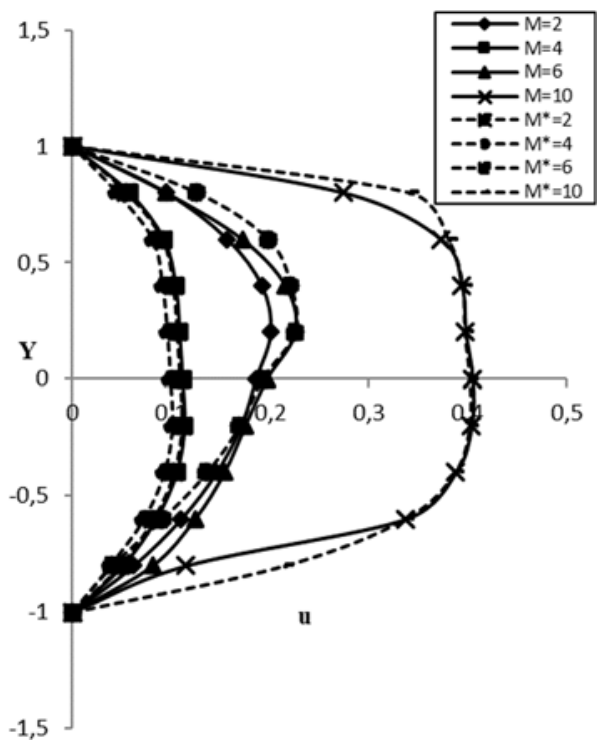

Fig.2. Primary velocity profiles $u_{1}, u_{2}$ (unsteady flow), $u_{1}^{*}, u_{2}^{*}$ (steady flow) for different $M$ and $m=2, \alpha=0.333, h=1, \sigma_{0}=2, \sigma_{01}=1.2, \sigma_{02}=1.5$, $\varepsilon=0.5, \rho=1, \omega=1, t=\pi / \omega \quad$ (non-conducting plates).

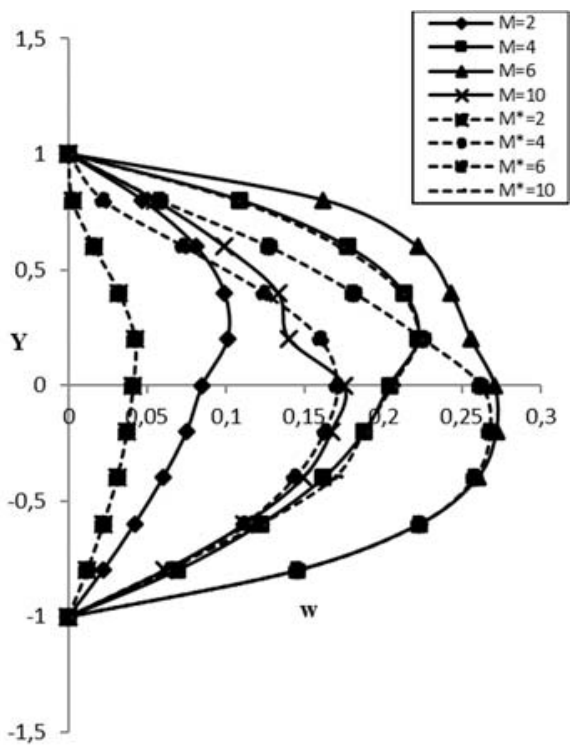

Fig.3. Secondary velocity profiles $w_{1}, w_{2}$ (unsteady flow), $w_{1}{ }^{*}, w_{2} *$ (steady flow) for different $M$ and $m=2, \alpha=0.333, h=1, s_{0}=2$, $\sigma_{01}=1.2, \quad \sigma_{02}=1.5, \quad \varepsilon=0.5, \quad \rho=1, \quad \omega=1$, $t=\pi / \omega$ (non-conducting plates). 


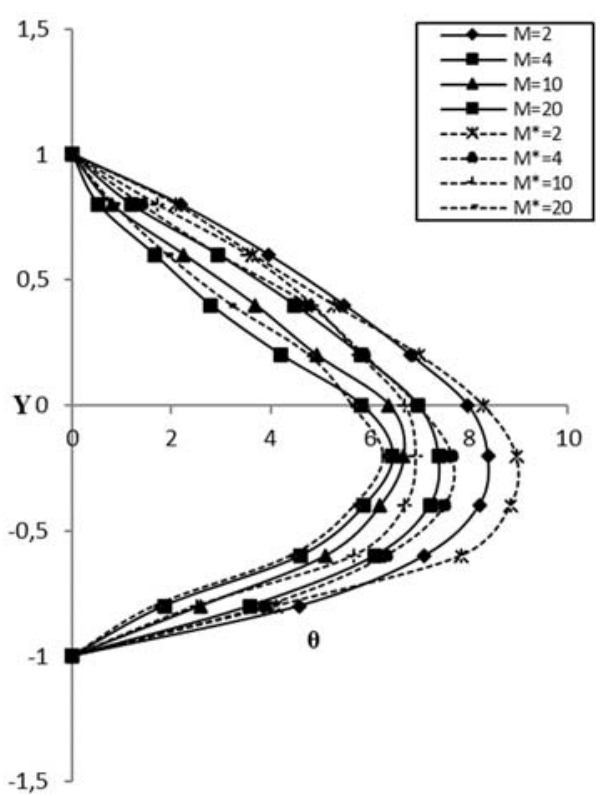

Fig.4. Temperature profiles $\theta_{1}, \theta_{2}$ (unsteady flow), $\theta_{1} *, \theta_{2} *$ (steady flow) for different $M$ and $m=2, \alpha=0.333, h=0.75, \sigma_{0}=1, \sigma_{01}=1.2$, $\sigma_{02}=1.5, \beta=0.5, \varepsilon=0.5, \rho=1, \omega=1, t=\pi / \omega$ (non-conducting plates).

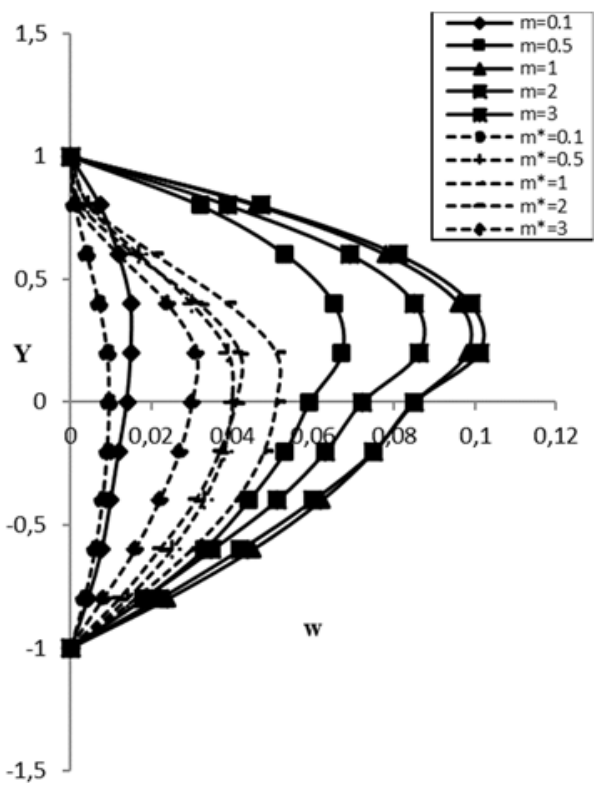

Fig.6. Secondary velocity profiles $w_{1}, w_{2}$ (unsteady flow), $w_{1}{ }^{*}, w_{2}{ }^{*}$ (steady flow) for different $m$ and $M=2, \quad \alpha=0.333, \quad h=1, \sigma_{0}=2, \sigma_{01}=1.2$, $\sigma_{02}=1.5, \quad \varepsilon=0.5, \quad \rho=1, \omega=1, t=\pi / \omega \quad$ (nonconducting plates).

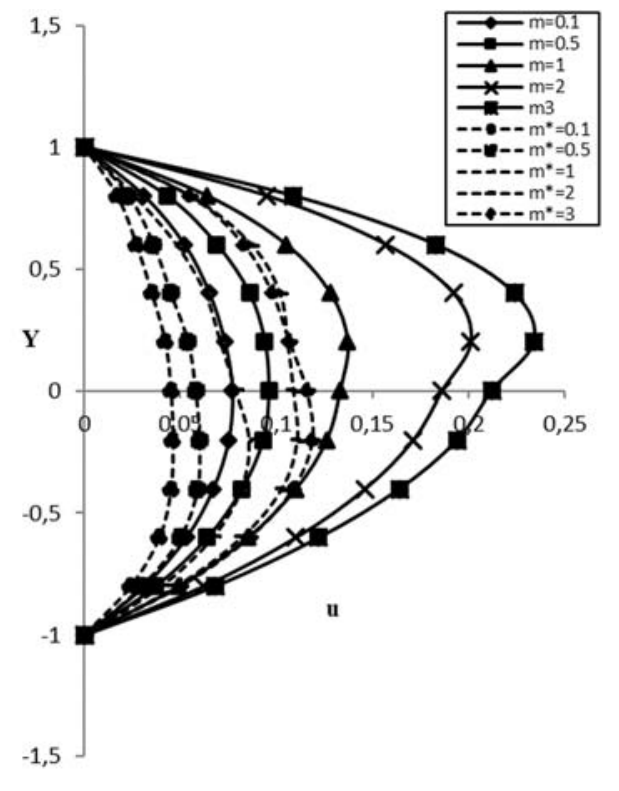

Fig.5. Primary velocity profiles $u_{1}, u_{2}$ (unsteady flow), $u_{1}^{*}, u_{2}^{*}$ (steady flow) for different ' $m$ ' and $\quad M=2, \alpha=0.333, h=1, \sigma_{0}=2, \sigma_{01}=1.2$, $\sigma_{02}=1.5, \varepsilon=0.5, \rho=1, \omega=1, t=\pi / \omega \quad$ (nonconducting plates).

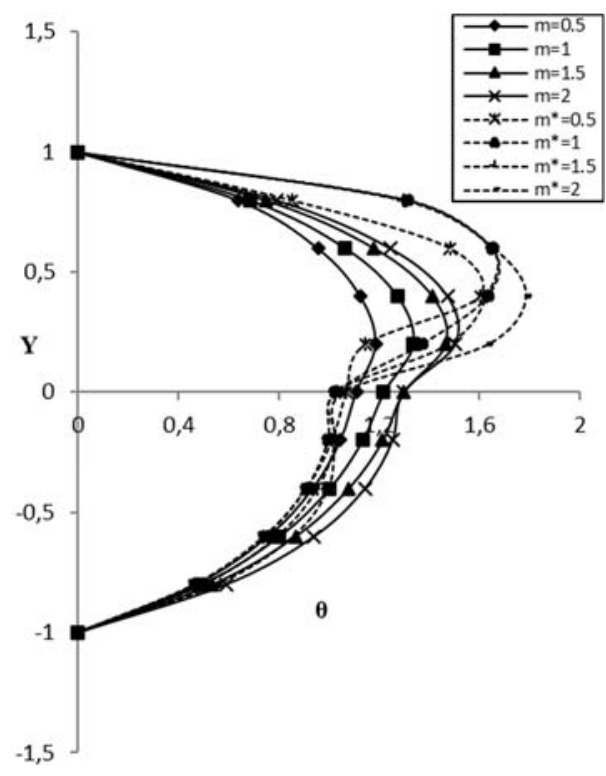

Fig.7. Temperature profiles $\theta_{1}, \theta_{2}$ (unsteady flow), $\theta_{1}^{*}, \theta_{2} *$ (steady flow) for different $\mathrm{m}$ and $M=4$, $\alpha=0.333, h=0.75, \sigma_{0}=1, \sigma_{01}=1.2, \sigma_{02}=1.5$, $\beta=0.5, \varepsilon=0.5, \rho=1, \omega=1, t=\pi / \omega \quad$ (nonconducting plates). 


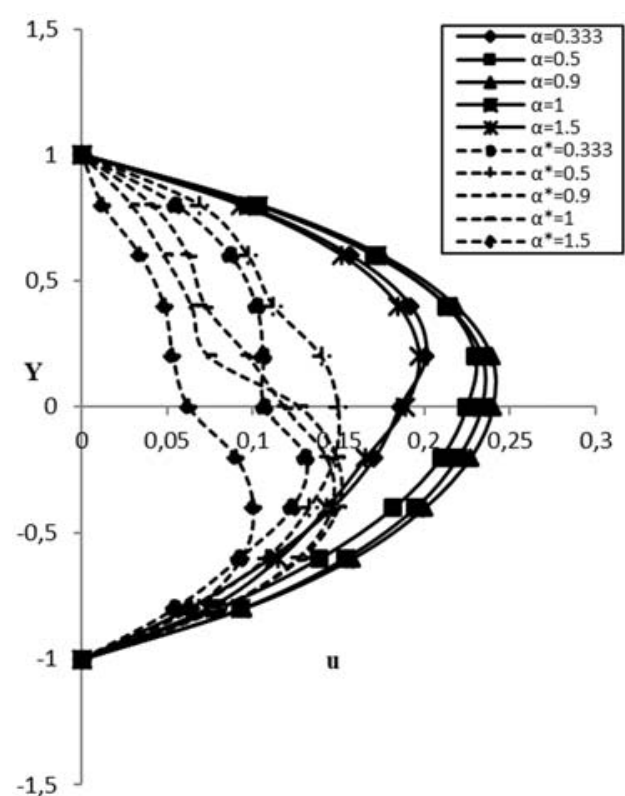

Fig.8. Primary velocity profiles $u_{1}, u_{2}$ (unsteady flow), $u_{1}^{*}, u_{2}{ }^{*}$ (steady flow) for different $\alpha$ and $M=2, \quad m=2, \quad h=1, \quad \sigma_{0}=2, \quad \sigma_{01}=1.2$, $\sigma_{02}=1.5, \quad \varepsilon=0.5, \quad \rho=1, \omega=1, t=\pi / \omega \quad$ (nonconducting plates).

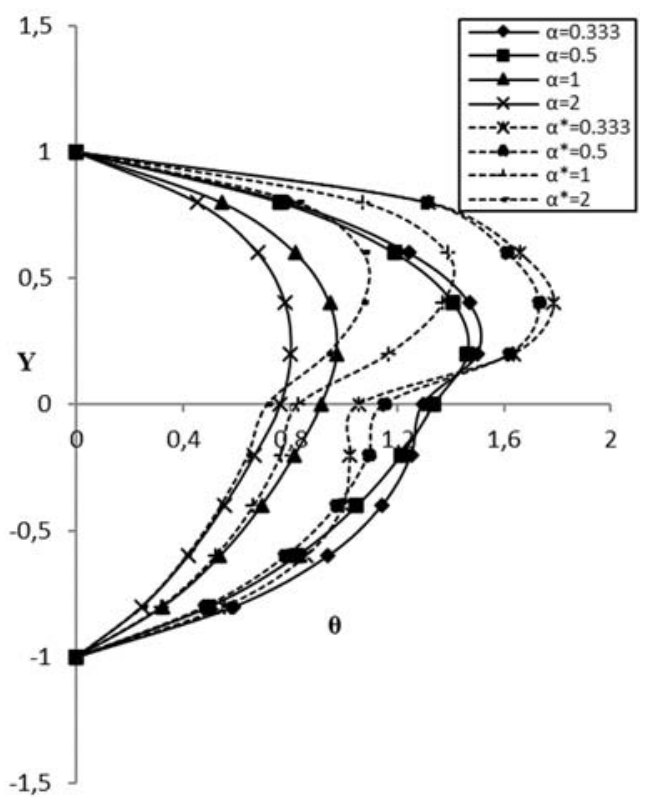

Fig.10. Temperature profiles $\theta_{1}, \theta_{2}$ (unsteady flow), $\theta_{1} *, \theta_{2} *$ (steady flow) for different $\alpha$ and $M=4$, $m=2, \quad h=0.75, \quad \sigma_{0}=1, \quad \sigma_{01}=1.2, \quad \sigma_{02}=1.5$, $\beta=0.5, \quad \varepsilon=0.5, \quad \rho=1, \omega=1, t=\pi / \omega \quad$ (nonconducting plates).

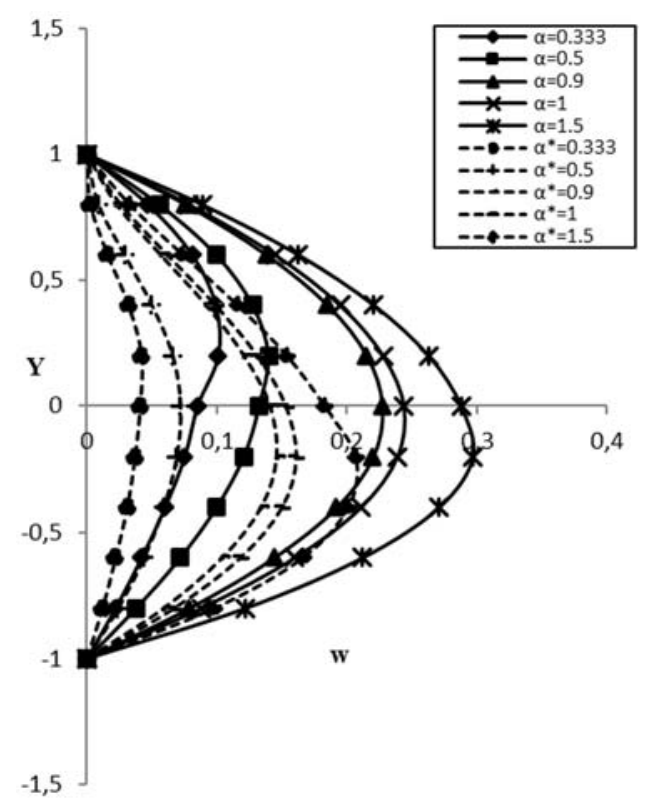

Fig.9. Secondary velocity profiles $w_{1}, w_{2}$ (unsteady flow), $w_{1}{ }^{*}, w_{2} *$ (steady flow) for different $\alpha$ and $M=2, \quad m=2, \quad h=1, \quad \sigma_{0}=2, \quad \sigma_{01}=1.2$, $\sigma_{02}=1.5, \quad \rho=1, \quad \omega=1, \quad t=\pi / \omega \quad$ (nonconducting plates).

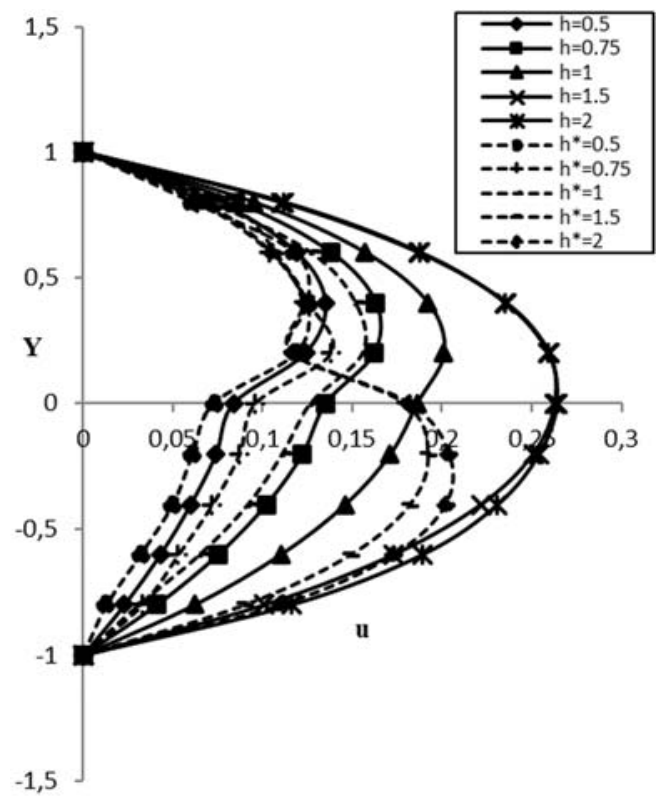

Fig.11. Primary velocity profiles $u_{1}, u_{2}$ (unsteady flow), $u_{1}^{*}, u_{2} *$ (steady flow) for different $\mathrm{h}$ and $M=2, \quad m=2, \quad \alpha=0.333, \quad \sigma_{0}=2, \quad \sigma_{01}=1.2$, $\sigma_{02}=1.5, \varepsilon=0.5, \rho=1, \omega=1, t=\pi / \omega \quad$ (nonconducting plates). 


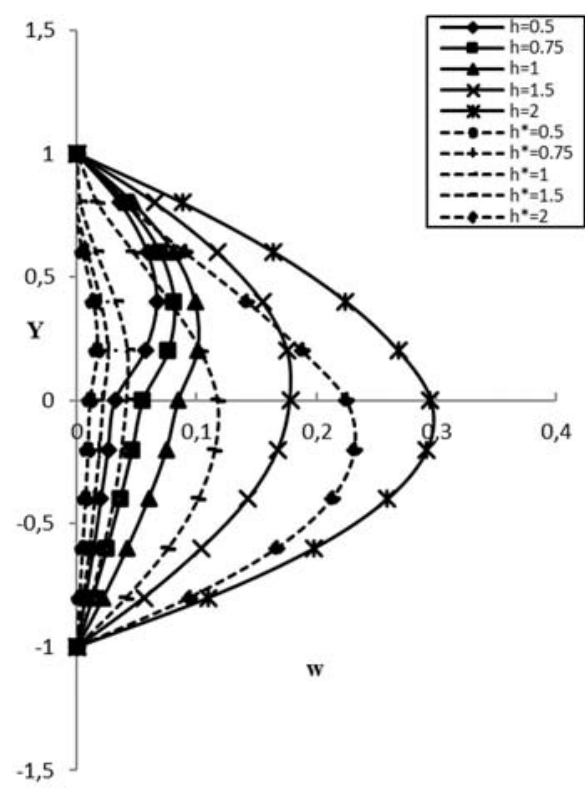

Fig.12. Secondary velocity profiles $w_{1}, w_{2}$ (unsteady flow), $w_{1}{ }^{*}, w_{2}{ }^{*}$ (steady flow) for different $h$ and $M=2, m=2, \alpha=0.333, \sigma_{0}=2$, $\sigma_{01}=1.2, \sigma_{02}=1.5, \varepsilon=0.5, \rho=1, \omega=1, t=\pi / \omega$ (non-conducting plates).

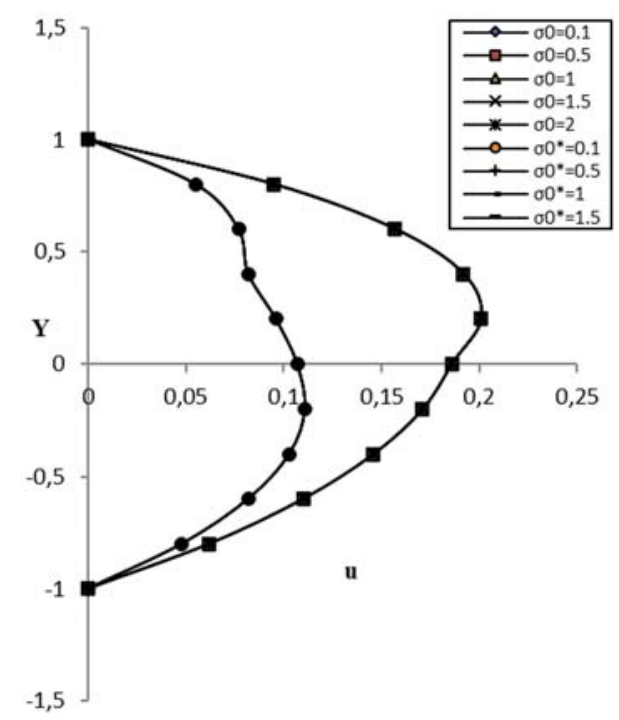

Fig.14. Primary velocity profiles $u_{1}, u_{2}$ (unsteady flow), $u_{1}^{*}, u_{2} *$ (steady flow) for different $\sigma_{0}$ and $M=2, \quad m=2, \quad \alpha=0.333, \quad h=1, \quad \sigma_{01}=1.2$, $\sigma_{02}=1.5, \varepsilon=0.5, \rho=1, \omega=1, t=\pi / \omega \quad$ (nonconducting plates).

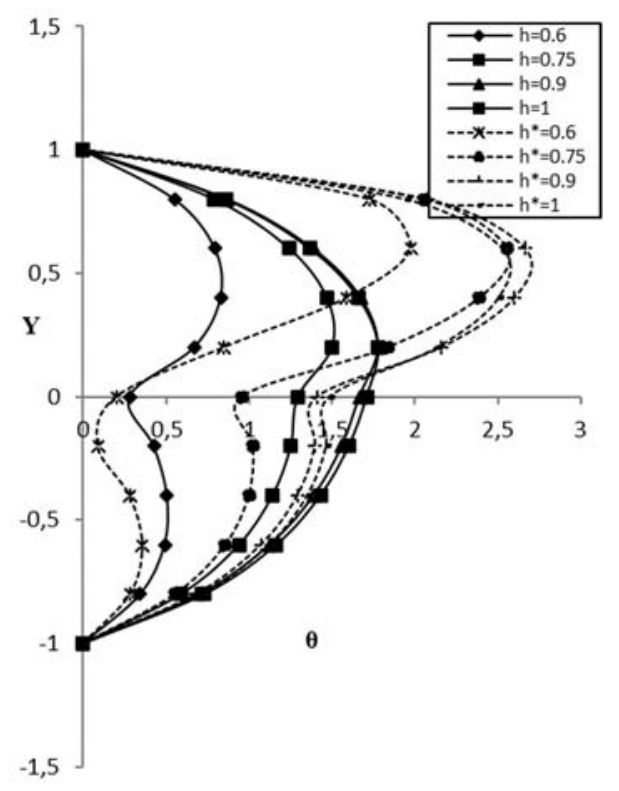

Fig.13. Temperature profiles $\theta_{1}, \theta_{2}$ (unsteady flow), $\theta_{1} *, \theta_{2} *$ (steady flow)for different $h$ and $M=4, \quad m=2, \quad \alpha=0.333, \sigma_{0}=1, \quad \sigma_{01}=1.2$, $\sigma_{02}=1.5, \varepsilon=0.5, \rho=1, \omega=1, t=\pi / \omega \quad$ (nonconducting plates).

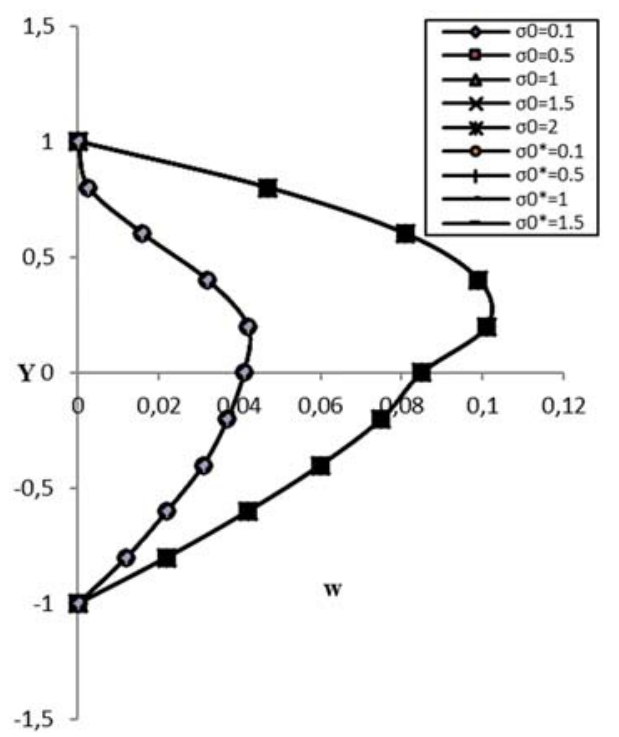

Fig.15. Secondary velocity profiles $w_{1}, w_{2}$ (unsteady flow), $w_{1}{ }^{*}, w_{2}{ }^{*}$ (steady flow) for different $\sigma_{0}$ and $M=2, m=2, \alpha=0.333, h=1$, $\sigma_{01}=1.2, \sigma_{02}=1.5, \varepsilon=0.5, \rho=1, \omega=1, t=\pi / \omega$ (non-conducting plates). 


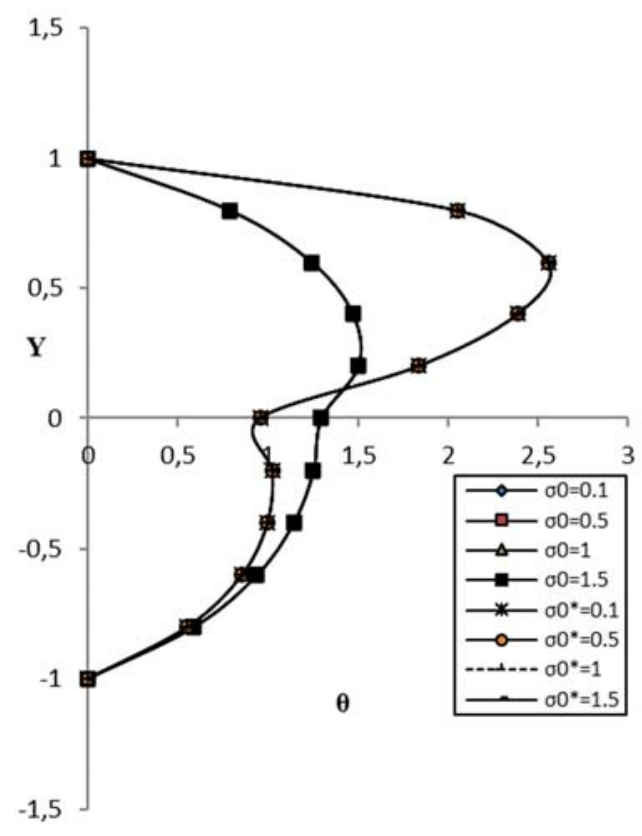

Fig.16. Temperature profiles $\theta_{1}, \theta_{2}$ (unsteady flow), $\theta_{1} *, \theta_{2} *$ (steady flow) for different $\sigma_{0}$ and $M=4$, $m=2, \alpha=0.333, h=0.75, \sigma_{01}=1.2, \sigma_{02}=1.5$, $\varepsilon=0.5, \rho=1, \omega=1, t=\pi / \omega \quad$ (non-conducting plates).

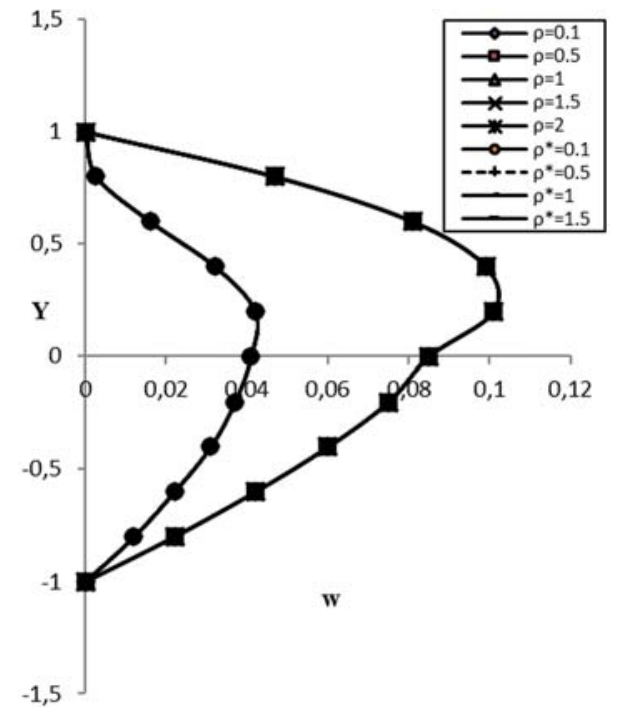

Fig.18. Secondary velocity profiles $w_{1}, w_{2}$ (unsteady flow), $w_{1} *, w_{2}{ }^{*}$ (steady flow) for different $\rho$ and $M=2, m=2, \alpha=0.333, \sigma_{0}=2$, $\sigma_{01}=1.2, \sigma_{02}=1.5, h=1, \varepsilon=0.5, \omega=1, t=\pi / \omega$ (non-conducting plates).

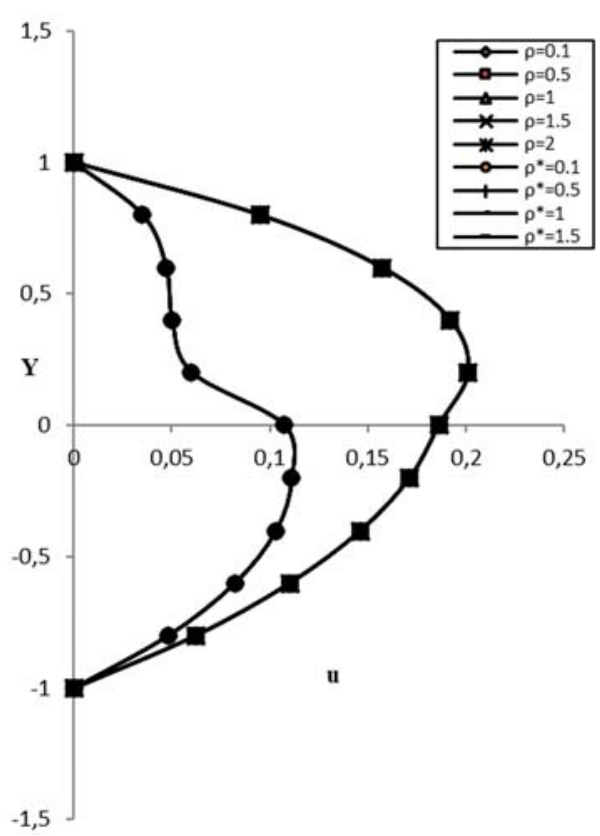

Fig.17. Primary velocity profiles $u_{1}, u_{2}$ (unsteady flow), $u_{1} *, u_{2} *$ (steady flow)for different $\rho$ and $M=2, \alpha=0.333, \sigma_{0}=2, \sigma_{01}=1.2, \sigma_{02}=1.5$, $h=1, \quad \varepsilon=0.5, \omega=1, t=\pi / \omega \quad$ (non-conducting plates).

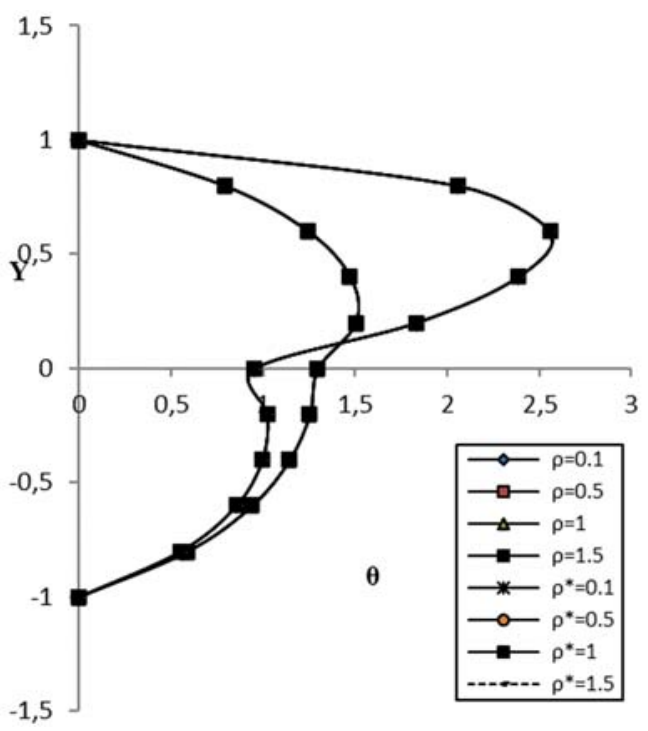

Fig.19. Temperature profiles $\theta_{1}, \theta_{2}$ (unsteady flow), $\theta_{1} *, \theta_{2} *$ (steady flow) for different $\rho$ and $M=4, \quad m=2, \quad \alpha=0.333, \quad h=0.75, \quad \sigma_{0}=2$, $\sigma_{01}=1.2, \sigma_{02}=1.5, \beta=1, \varepsilon=0.5, \omega=1, t=\pi / \omega$ (non-conducting plates). 


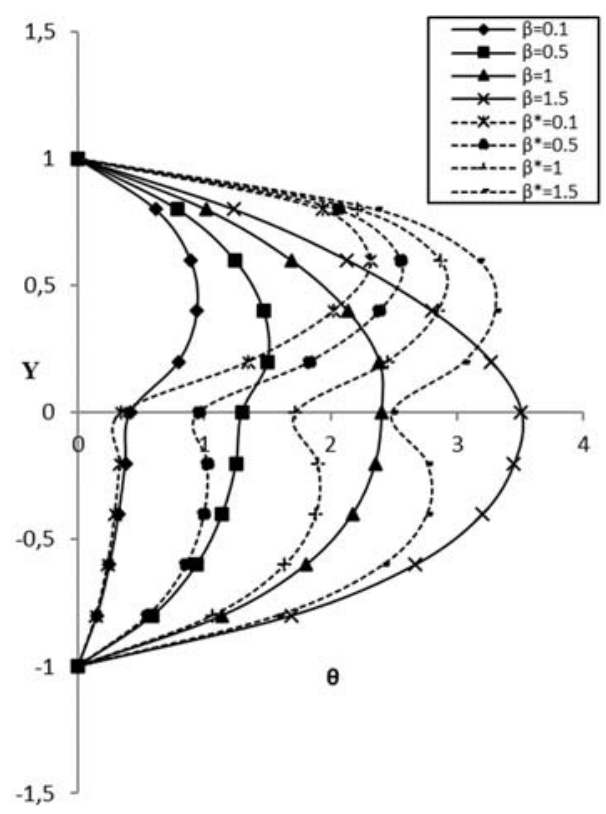

Fig.20. Temperature profiles $\theta_{1}, \theta_{2}$ (unsteady flow), $\theta_{1}^{*}, \theta_{2} *$ (steady flow) for different $\beta$ and $M=4, m=2$, $\alpha=0.333, h=0.75, \sigma_{0}=1, \sigma_{01}=1.2, \sigma_{02}=1.5, \varepsilon=0.5, \rho=1, \omega=1, t=\pi / \omega$ (non-conducting plates).

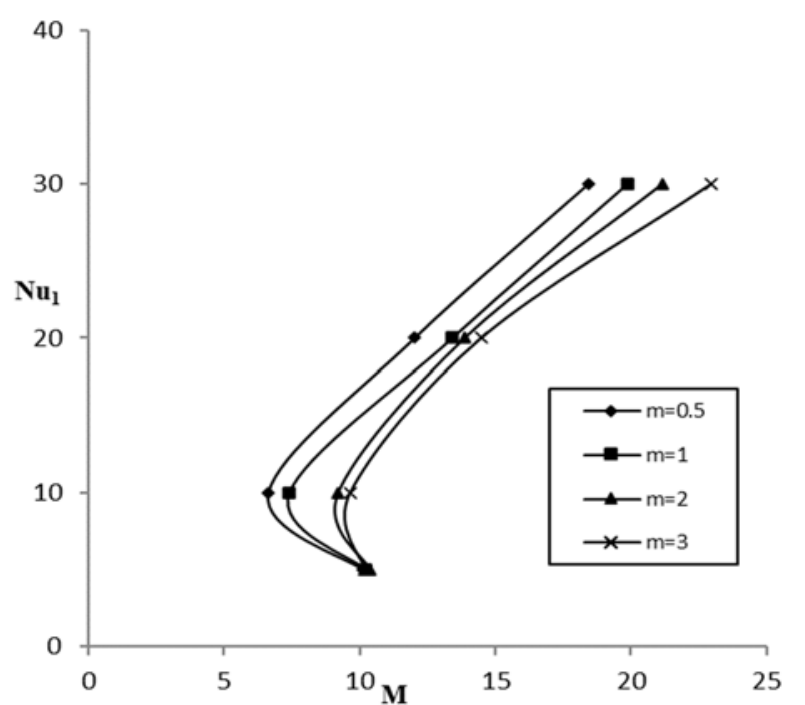

Fig.21. Nusselt Number $\left(N u_{1}\right)$ for different $M$ and $\alpha=0.333, \sigma_{0}=1, \sigma_{01}=1.2, \sigma_{02}=1.5, h=0.75$, $\beta=0.5, \quad \varepsilon=0.5, \quad \rho=1, \omega=1, t=\pi / \omega \quad$ (nonconducting plates).

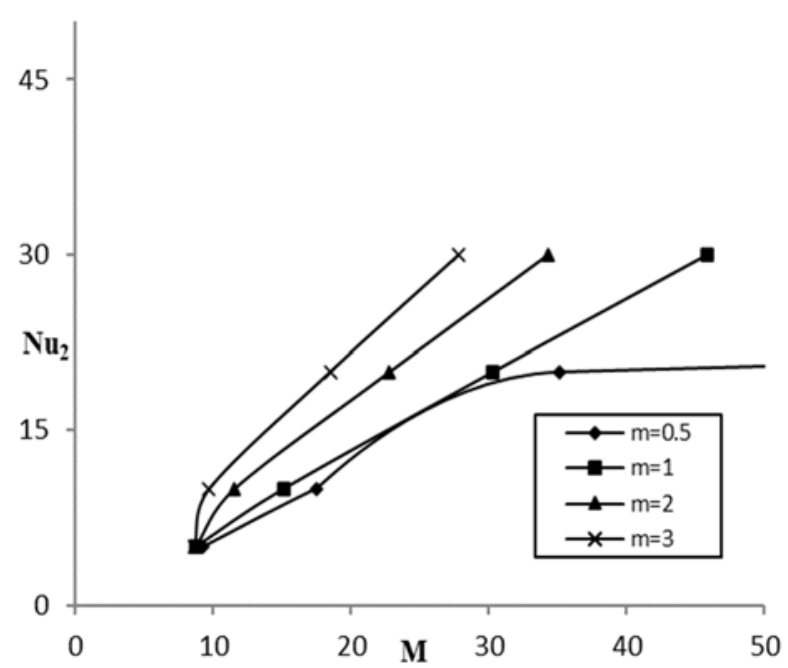

Fig.22. Nusselt Number $\left(N u_{2}\right)$ for different $M$ and $\alpha=0.333, \sigma_{0}=1, \sigma_{01}=1.2, \sigma_{02}=1.5, h=0.75$, $\beta=0.5, \quad \varepsilon=0.5, \quad \rho=1, \quad \omega=1, t=\pi / \omega \quad$ (nonconducting plates)

The solid lines in the figures describe the velocity and temperature profiles for unsteady motions and dash-spot lines for the steady motions. The effect of flow parameters, like the Hartmann number $M$, Hall parameter ' $m$ ', density ratio $\rho$, viscosity ratio $\alpha$, height ratio ' $h$ ', electrical conductivity ratio $\sigma_{0}$ and thermal conductivity ratio $\beta$ on the velocity and temperature fields is discussed. Since the problem involves a number of 
non-dimensional parameters, so for simplicity, we fix the parameters, namely $\sigma_{01}=1.2$ and $\sigma_{02}=1.5$ in all the numerical calculations and the effect of other important parameters is investigated.

We observe that the solutions are independent of ' $s$ ' (ratio of the electron pressure to the total pressure). It is found that this investigation is in great concurrence with that of Raju [40] when the motion is under steadystate. Also, this study agrees with the solutions of Malashetty and Leela [24] where there are no effects of Hall currents in their investigation for a steady magnetohydrodynamic(MHD) two-fluid flow.

The effect of varying the Hartmann number ' $M$ ' on the velocity distribution in the two regions is shown in Figs. 2 and 3. It is seen from Fig.2 that an increase in the Hartmann number ' $M$ ' up to the estimation of $M=4$ diminishes the primary velocity distribution and past this estimation it increases in the two regions. While from Fig. 3, it is discovered that when there is an increase in the Hartmann number ' $M$ ' up to the estimation of $M=6$, the secondary velocity distribution increases and past this estimation it diminishes in the two regions. However, it is seen that the greatest primary and secondary velocity distributions in the channel tend to move over the channel centerline toward the region-I as ' $M$ ' increases. The effect of varying the Hartmann number ' $M$ ' on temperature distribution is displayed in Fig.4. It is discovered that an increase in Hartmann number ' $M$ ' diminishes the temperature distribution in the two-fluid regions. The greatest temperature in the channel tends to move below the channel focus line towards the region-II as ' $M$ ' increases at the point when the remaining parameters are fixed. It is clear from these observations that the magnetic field has a more noticeable effect on velocity distributions than the temperature distributions.

The effect of changeable Hall parameter ' $m$ ' on both primary and secondary velocity distributions appears in Figs.5 and 6. It is noticed from Fig. 5 that a rise in the Hall parameter increases the primary velocity distribution in the two regions. This implies that the Hall parameter accelerates velocity of the fluids in the primary fluid flow direction. But, it is evident from Fig. 6 that an increase in ' $m$ ' up to the estimation of $m=2$ enhances the secondary velocity distribution and past this estimation it diminishes in the region-I. However, in the region-II, an increase in ' $m$ ' up to the estimation of $m=1$ increases and from that point diminishes. This kind of tendency may be due to the minimum retarding force acting along the y-direction that is created due to the interaction of the applied magnetic field and the Hall current in electrically conducting fluids. So the newness of the Hall current shown in this study is that it not only induces a cross flow (i.e., secondary flow) transverse to the main flow(primary flow) but also makes it oscillatory. Likewise, it is observed that the greatest primary and secondary velocity distributions in the channel tend to move over the channel centerline towards the region I as ' $m$ ' increases. The cause of the variable Hall parameter ' $m$ ' on temperature distribution is shown in Fig.7. It is seen in two regions that the temperature distribution increases with an increase in the Hall parameter ' $m$ '. The most extreme temperature distribution in the channel tends to move over the channel centerline towards the region-I as the Hall parameter ' $m$ ' increases.

The outcome of $\alpha$, that is, the ratio of viscosities of the two fluids is shown in Figs. 8 and 9. It is evident from Fig. 8 that an increase of $\alpha$ up to the estimation $\alpha=0.9$ rises the primary velocity distribution and from there on diminishes in the two regions. It is observed from Fig. 9 that an increase in $\alpha$ increases the secondary velocity distribution in the two regions. The greatest primary velocity distribution in the channel tends to move above the channel centerline towards the region-I as $\alpha$ increases and the most extreme secondary velocity distribution in the channel tends to move beneath the channel focus line towards the region-II as $\alpha$ increases. The outcome of the viscosity ratio $\alpha$ on the temperature distribution is shown in Fig.10. It is observed that a rise in $\alpha$ diminishes the temperature distribution in the two regions. Moreover, the temperature distribution in the channel tends to move over the channel centerline towards the region-I as $\alpha$ increases.

The significance of the variance of the height ratio ' $h$ ' on both primary and secondary velocity distributions is illustrated in Figs. 11 and 12. It is seen that an increase in ' $h$ ' increases both primary and secondary velocity distributions in the two regions. The most extreme primary and secondary velocity distributions in the channel tend to move over the channel focus line toward the region-I as ' $h$ ' increases. The impact of changing the height ratio ' $h$ ' on the temperature distribution is shown in Fig.13. It is seen in the region-I that an increase in ' $h$ ' enhances the temperature distribution up to $h=0.9$ and from there on 
diminishes, but the temperature increases in region-II. The most extreme temperature in the channel tends to move over the channel centerline towards the region-I as ' $h$ ' increases.

The impact of the electrical conductivity ratio $\sigma_{0}$ is shown in Figs.14 and 15. It is discovered that there is an insignificant variation on both primary and secondary velocity distributions as $\sigma_{0}$ increases. The maximum primary and secondary velocity distributions in the channel tend to move over the channel focus line toward the region-I as $\sigma_{0}$ increases. The effect of varying the electrical conductivity ratio $\sigma_{0}$ on the temperature distribution is shown in Fig.16. It is discovered that there is no minor departure from the temperature distribution in the two regions as $\sigma_{0}$ increases. The maximum temperature distribution in the channel tends to move over the channel centerline towards the region-I as $\sigma_{0}$ increases.

The effect of $\rho$, that is the ratio of densities of the two fluids is shown in Figs.17 and 18. It is found that there is no significant variation in both primary and secondary velocity distributions as $\rho$ increases. The most extreme primary and secondary velocity distributions in the channel tend to move over the channel centerline toward the region-I as $\rho$ increases. Figure 19 shows the effect of $\rho$ on the temperature distribution in the two-fluid regions. It is discovered that there is not much critical departure from the temperature distribution in the two regions as $\rho$ increases. The greatest temperature in the channel tends to move over the channel focus line towards the region $-\mathrm{I}$ as $\rho$ increases.

The effect of the thermal conductivity ratio $\beta$ on the temperature distribution is exhibited in Fig.20. It is observed that an increase in $\beta$ is to build temperature distribution in the two regions. The greatest temperature in the channel tends to move over the channel centerline towards the region-I as $\beta$ increases. This indicates that the thermal conductivity ratio accelerates the fluids temperature towards the region-I.

The heat transfer coefficient rates against the Hartmann number are shown in Figs.21 and 22. It is discovered that the rate of heat transfer coefficient increases as ' $M$ ' increases at the point when the remaining parameters are fixed. It is also found that an increase in the Hall parameter ' $M$ 'up to specific estimation diminishes the rate of the heat transfer coefficient and past this estimation increases the rate of heat transfer coefficient at the upper plate, while the rate of heat transfer coefficient increases at the lower plate as the Hall parameter increases exactly when all the other governing parameters are fixed.

\section{Conclusions}

A novel investigation of unsteady magnetohydrodynamic(MHD) heat transfer two-fluid flow of ionized gases driven by a constant pressure gradient through a horizontal channel bounded by two parallel, non-conducting plates under the influence of an applied transverse magnetic field with Hall effect is made. The equations of motion, current and energy for the two-fluid flow regions are detailed. The resulting partial differential equations are solved analytically by employing the regular first order perturbation method and the solutions for both velocity and temperature distributions in the two-fluid regions are obtained. The heat transfer rates at the plates are also expressed in the form of a non-dimensional Nusselt number. The effects of flow parameters such as, the Hartmann number, the Hall parameter, the ratios of viscosity, density, height, electrical conductivity and thermal conductivity on velocity and temperature fields are analyzed graphically. The main findings of this study are summarized as follows:

- An increase in the Hartmann number diminishes the primary velocity distribution up to a specific estimation of the parameter and from that point the velocity increases. Yet, the secondary velocity distribution increases up to a specific estimation of the parameter and from that point diminishes as the Hartmann number increases.

- An increase in the Hall parameter improves the primary velocity profile, while the secondary velocity distribution increases up to a specific estimation of the parameter and from that point diminishes.

- A rise in the viscosity ratio raises the primary velocity distribution up to a specific estimation of the parameter and from that point diminishes. However, the secondary velocity distribution increases. 
- An increase in height ratio raises the primary and secondary velocity distributions.

- An increase in the Hartmann number and viscosity ratio reduces the temperature distribution.

- An increase either in the Hall parameter or thermal conductivity ratio enhances the temperature distribution.

- An increase in height ratio up to a specific estimation increases the temperature distribution and from that point diminishes.

- Most of the extreme temperature in the channel, tends to move above the channel centerline towards the region-I at the point when each one of the parameters increases except for the Hartmann number, while the most extreme temperature in the channel tends to move below the channel centerline towards the region-II as the Hartmann number increases.

- The rates of heat transfer coefficient at the upper and lower plates increase as the Hartmann number increases.

- An increase in the Hall parameter up to a specific estimation diminishes the rate of heat transfer coefficient at the upper plate and past this estimation it increases, while the rate of heat transfer coefficient increases at the lower plate as the Hall parameter increases.

\section{Nomenclature}

$$
\begin{aligned}
& \left.\begin{array}{l}
A_{1}, A_{2}, \ldots a_{1}, a_{2}, \ldots \\
B_{1}, B_{2}, \ldots b_{1}, b_{2}, \ldots \\
D_{1}, D_{2}, D_{3} .
\end{array}\right\}- \text { symbols/or functional relations considered in the equations and solutions } \\
& B_{0} \quad-\text { applied uniform transverse magnetic field } \\
& \bar{B} \text { - magnetic flux density } \\
& D_{1}, D_{2}, D_{3}-\text { symbols represented as } D_{1}=\beta_{1}+i \beta_{2}, D_{2}=\beta_{3}+i \beta_{4}, D_{3}=i \sigma_{1}+m \sigma_{2} \\
& e-\text { Electric charge } \\
& E_{x}, E_{z} \text { - applied electric fields in } x \text { - and } z \text { - directions respectively } \\
& h \text { - ratio of the heights of the two regions } \\
& h_{1} \text { - height of the channel in the upper region (Region-I) } \\
& h_{2} \quad-\text { height of the channel in the lower region (Region-II) } \\
& I_{x_{1}}, I_{z_{1}} ; I_{x_{2}}, I_{z_{2}} \quad \text { - dimensionless current densities along } x \text { - and } z \text {-directions in two fluid regions } \\
& J_{x}, J_{z} \text { - current densities in } x \text { - and } z \text {-directions, respectively } \\
& \bar{J} \text { - current density } \\
& K_{1}, K_{2}-\text { thermal conductivities of the two fluids in region-I and region-II } \\
& m \text { - Hall parameter } \\
& m_{1}, m_{2}, m_{3}, \cdots-\text { symbols/or functional relations considered in the equations and solutions } \\
& m_{x}, m_{z}-\text { dimensionless electric fields } \\
& M \quad \text { - Hartmann number } \\
& N_{1}, N_{2} \text { - symbols represented as } N_{1}=m_{x_{1}}+i m_{z_{1}} \text { and } N_{2}=m_{x_{2}}+i m_{z_{2}} \\
& \mathrm{Nu}_{1}, \mathrm{Nu}_{2}-\text { rate of heat transfer coefficients at upper and lower plates respectively } \\
& P \text { - pressure } \\
& p_{e}-\text { electron pressure } \\
& q_{01}, q_{02}, q_{11}, q_{12} \text { - complex notations represented for simplicity } \\
& q_{m_{1}}, q_{m_{2}} \text { - complex form of the mean velocities } \\
& \text { Pr - Prandtl number } \\
& \mathrm{s}-=p e / p \text { (ratio of electron pressure to the total pressure) } \\
& t, T \text { - time, temperature }
\end{aligned}
$$




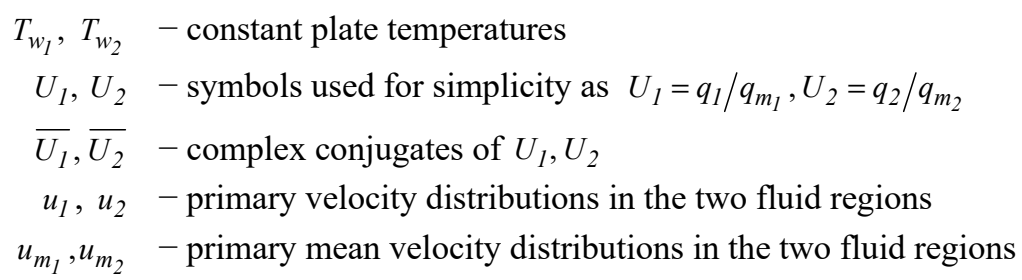

$u_{01}(y), u_{02}(y)$ - primary velocity distributions in the steady state case in two fluid regions

$u_{11}(y), u_{12}(y)$ - transient primary velocity components in the two fluid regions

$u_{p}--\frac{\partial p}{\partial x} \frac{h_{1}^{2}}{\mu_{l}}$, characteristic velocity

$w_{1}, w_{2}-$ secondary velocity distributions in the two fluid regions

$w_{m_{1}}, w_{m_{2}}-$ secondary mean velocity distributions in the two fluid regions

$w_{01}(y), w_{02}(y)$ - secondary velocity distributions in the steady state case in two regions

$w_{11}(y), w_{12}(y)-$ transient secondary velocity components in two regions

$(x, y, z)$ - space co-ordinates

$\alpha-$ ratio of viscosities

$\beta-$ ratio of thermal conductivities

$\mu_{1}, \mu_{2}-$ viscosities of the two fluids

$\sigma_{01}, \sigma_{02}$ - electrical conductivities of the two fluids

$\sigma_{0}-$ ratio of electrical conductivities

$\sigma_{11}, \sigma_{12}, \sigma_{21}, \sigma_{22}-$ modified conductivities parallel and normal to the direction of electric filed

$\varepsilon \quad-$ amplitude (a small constant quantity, $\varepsilon<<1$ )

$\rho-$ ratio of densities

$\rho_{0} \quad$ - free charge density

$\rho_{1}, \rho_{2}-$ densities of the two fluids

$\theta_{1}, \theta_{2}$ - non-dimensional forms of temperature distributions of the two fluids

$\theta_{01}(y), \theta_{02}(y)$ - temperature distributions under the steady state in the two regions

$\theta_{11}(y), \theta_{12}(y)$ - temperature distributions under transient state in the two fluid regions

$\tau, \tau_{e}-$ mean collision time between electron and ion, electron and neutral particles

$\omega$ - frequency of oscillation

$\sigma_{1}, \sigma_{2}-$ modified conductivities parallel and normal to the direction of electric field

\section{Appendix}

$$
\begin{aligned}
& \beta_{1}=1-s\left(\frac{1}{1+m^{2}}\right), \quad \beta_{2}=\frac{-s m}{1+m^{2}}, \quad \beta_{3}=1-s\left(1-\frac{\sigma_{0} \sigma_{01}}{1+m^{2}}\right), \quad \beta_{4}=\frac{-s \sigma_{0} \sigma_{02} m}{1+m^{2}}, \\
& D_{1}=\beta_{1}+i \beta_{2}, \quad D_{2}=\beta_{3}+i \beta_{4}, \quad N_{1}=m_{1 x}+i m_{1 z}, \quad N_{2}=m_{2 x}+i m_{2 z}, \\
& D_{3}=i \sigma_{1}+m \sigma_{2}, \quad a_{1}=\frac{M^{2}}{1+m i}, \quad a_{2}=-\left(\mathrm{D}_{1}+\frac{M^{2} N_{1}}{m-i}\right), \quad a_{3}=\frac{\alpha h^{2} M^{2} D_{3}}{1+m^{2}}, \\
& a_{4}=-\left(D_{2} \alpha h^{2}+\frac{\alpha h^{2} M^{2} N_{2} D_{3}}{1+m^{2}}\right), \quad A_{1}=a_{5} a_{8}+a_{11} a_{9}, \quad A_{3}=\frac{a_{8}+A_{1}\left(1-e^{m_{1}-m_{2}}\right)}{1-e^{-m_{3}+m_{4}}},
\end{aligned}
$$




$$
\begin{aligned}
& A_{2}=\frac{e^{m_{2}} D_{1}+M^{2} N_{1}(i+m)}{M^{2}(m i-1)}-A_{1} e^{m_{1}-m_{2}}, \quad A_{5}=\frac{e^{-m_{2}} D_{1}(m i+1)}{M^{2}}, \quad A_{8}=\frac{-m e^{-m_{2}}}{(m i-1)}, \\
& A_{4}=\frac{-e^{m_{4}}\left\{D_{2} \alpha h^{2}\left(1+m^{2}\right)+\alpha h^{2} M^{2} N_{2} D_{3}\right\}}{\alpha h^{2} M^{2} D_{3}}-A_{3} e^{-m_{3}+m_{4}}, \quad m_{5}=\sqrt{\left(a_{1}-\omega \tan \omega t\right)}, \\
& A_{6}=\frac{e^{-m_{6}}\left\{M^{2}+e^{m_{2}+m_{5}} D_{1}(m i+1)\right\}}{M^{2}}, \quad m_{6}=-\sqrt{\left(a_{1}-\omega \tan \omega t\right)}, \quad m_{3}=\sqrt{a_{3}}, \\
& A_{9}=\frac{\varepsilon \cos \omega t\left\{a_{8} m_{6}\left(e^{m_{5}}-1\right)+\left(m_{3} m_{5}-m_{4} m_{5} e^{-m_{3}+m_{4}}\right)\left(e^{m_{6}}-1\right)\right\}}{\alpha h m_{5} m_{6}}, \quad m_{1}=\sqrt{a_{1}}, \\
& A_{10}=\frac{\varepsilon \cos \omega t\left\{a_{9} m_{8}\left(e^{m_{7}}-1\right)+m_{1}-m_{2} m_{7} e^{m_{1}-m_{2}}\left(e^{m_{8}}-1\right)\right\}}{m_{7} m_{8}}, \quad m_{2}=-\sqrt{a_{1}}, \\
& m_{4}=-\sqrt{a_{3}}, \quad m_{7}=\sqrt{\left(a_{3}-\omega \tan \omega t\right)}, \quad m_{8}=-\sqrt{\left(a_{3}-\omega \tan \omega t\right)}, \quad B_{1}=b_{61}, \\
& B_{2}=-b_{53}-b_{61}, \quad B_{3}=b_{60}, \quad B_{4}=-b_{54}+b_{60}, \quad B_{5}=-b_{116}\left(e^{2 \sqrt{\omega \tan \omega t}}\right)+b_{112}, \\
& B_{6}=b_{116}, \quad B_{7}=-b_{117}\left(e^{2 \sqrt{\omega \tan \omega t}}\right)+b_{113}, \quad B_{8}=b_{117}, b_{18}=\frac{b_{10}}{m_{1}+\overline{m_{1}}} \\
& b_{19}=\frac{-\left\{\left(1+m^{2}\right) m_{1} \overline{m_{2}}+M^{2}\right\}\left(A_{1} \overline{A_{2}}\right)\left(m_{1}+\overline{m_{2}}\right)^{2}}{\left(1+m^{2}\right) q_{m_{l}} \overline{q_{m_{l}}}}, \quad b_{22}=\frac{M^{2} m_{1}^{2} A_{1}\left(\overline{a_{2}}+\overline{q_{m_{l}}} \overline{a_{1}}\right)}{\left(1+m^{2}\right) q_{m_{l}} \overline{q_{m_{l}}} \overline{a_{1}}}, \\
& b_{20}=\frac{-\left\{\left(1+m^{2}\right) m_{2} \overline{m_{1}}+M^{2}\right\}\left(A_{2} \overline{A_{l}}\right)\left(m_{2}+\overline{m_{l}}\right)^{2}}{\left(1+m^{2}\right) q_{m_{l}} \overline{q_{m_{l}}}}, \quad b_{23}=\frac{M^{2} m_{2}^{2} A_{2}\left(\overline{a_{2}}+\overline{q_{m_{l}}} \overline{a_{1}}\right)}{\left(1+m^{2}\right) q_{m_{l}} \overline{q_{m_{l}}} \overline{a_{l}}}, \\
& b_{21}=\frac{\left.-\left\{\left(1+m^{2}\right)\right) m_{2} \overline{m_{2}}+M^{2}\right\}\left(A_{2} \overline{A_{2}}\right)\left(m_{2}+\overline{m_{2}}\right)^{2}}{\left(1+m^{2}\right) q_{m_{l}} \overline{q_{m_{l}}}}, \quad b_{24}=\frac{M^{2}{\overline{A_{1}}}_{m_{1}}^{2}\left(a_{2}+q_{m_{1}} a_{1}\right)}{\left(1+m^{2}\right) a_{1} q_{m_{l}} \overline{q_{m_{l}}}} \\
& b_{25}=\frac{M^{2}{\overline{A_{2} m_{2}}}^{2}\left(a_{2}-q_{m_{l}} a_{1}\right)}{\left(1+m^{2}\right) a_{1} q_{m_{l}} \overline{q_{m_{l}}}}, \quad b_{26}=\frac{-2 M^{2}\left\{\overline{a_{2}}\left(a_{2}+a_{1} q_{m_{l}}\right)+a_{1} \overline{a_{1}}\left(\overline{q_{m_{l}}} a_{2}+q_{m_{l}} \overline{q_{m_{l}}}\right)\right\}}{\left(1+m^{2}\right) a_{1} \overline{a_{1}} q_{m_{l}} \overline{q_{m_{l}}}} \\
& b_{44}=\frac{-\beta \overline{A_{3}}\left(m_{3}+\overline{m_{3}}\right)^{2}\left\{A_{3} m_{3} \overline{m_{3}}\left(1+m^{2}\right)+M^{2} h^{2} \alpha \sigma_{1} A_{3}\right\}}{\alpha\left(1+m^{2}\right)\left(q_{m_{2}} \overline{q_{m_{2}}}\right)},
\end{aligned}
$$




$$
\begin{aligned}
& b_{45}=\frac{-\beta A_{3} \overline{A_{4}}\left(m_{3}+\overline{m_{4}}\right)^{2}\left\{m_{3}\left(1+m^{2}\right) \overline{m_{4}}+M^{2} h^{2} \alpha \sigma_{1}\right\}}{\alpha\left(1+m^{2}\right)\left(q_{m_{2}} \overline{q_{m_{2}}}\right)}, \\
& b_{46}=\frac{-\beta A_{4} \overline{A_{3}}\left(m_{4}+\overline{m_{3}}\right)^{2}\left\{m_{4} \overline{m_{3}}\left(1+m^{2}\right)+M^{2} h^{2} \alpha \sigma_{1}\right\}}{\alpha\left(1+m^{2}\right)\left(q_{m_{2}} \overline{q_{m_{2}}}\right)}, \\
& b_{47}=\frac{-\beta A_{4} \overline{A_{4}}\left(m_{4}+\overline{m_{4}}\right)^{2}\left\{\overline{m_{4}} m_{4}\left(1+m^{2}\right)+M^{2} h^{2} \alpha \sigma_{1}\right\}}{\alpha\left(1+m^{2}\right)\left(q_{m_{2}} \overline{q_{m_{2}}}\right)}, \\
& b_{48}=\frac{M^{2} A_{3} m_{3}^{2} \beta \sigma_{1} h^{2}\left(\overline{a_{4}}+\overline{q_{m_{2}}} \overline{a_{3}}\right)}{\left(1+m^{2}\right)\left(q_{m_{2}} \overline{q_{m_{2}}}\right) \overline{a_{3}}}, \quad b_{49}=\frac{M^{2} h^{2} m_{4}^{2} A_{4} \beta \sigma_{1}\left(\overline{a_{4}}+\overline{q_{m_{2}}} \overline{a_{3}}\right)}{\left(1+m^{2}\right)\left(q_{m_{2}} \overline{q_{m_{2}}}\right) \overline{a_{3}}}, \\
& b_{50}=\frac{M^{2} h^{2}{\overline{A_{3}}}^{2}{ }^{2} \beta \sigma_{1}\left(a_{4}-a_{3} q_{m_{2}}\right)}{\left(1+m^{2}\right)\left(q_{m_{2}} \overline{q_{m_{2}}}\right) a_{3}}, \quad b_{51}=\frac{M^{2} h^{2}{\overline{A_{4}}}_{m_{4}}^{2} \beta \sigma_{1}\left(a_{4}+q_{m_{2}} a_{3}\right)}{\left(1+m^{2}\right)\left(q_{m_{2}} \overline{q_{m_{2}}}\right) a_{3}}, \\
& b_{52}=\frac{-2 M^{2} h^{2} \beta \sigma_{1}}{\left(1+m^{2}\right)\left(q_{m_{2}} \overline{q_{m_{2}}}\right)}\left\{\frac{a_{4}\left(\overline{a_{4}}+\overline{q_{m_{2}}} \overline{a_{3}}\right)}{a_{3} \overline{a_{3}}}+q_{m_{2}}\left(\frac{\overline{a_{4}}}{\overline{\overline{a_{3}}}}+\overline{q_{m_{2}}}\right)\right\}, \\
& b_{70}=\frac{-\varepsilon^{3} \cos ^{3} \omega t \overline{A_{5}}\left\{M^{2} A_{5}+A_{5} m_{5} \overline{m_{5}}\left(1+m^{2}\right)\right\}}{\left(1+m^{2}\right) q_{m_{l}} \overline{q_{m_{l}}}\left\{\left(m_{5}+\overline{m_{5}}\right)^{2}+(\omega \tan \omega t)\right\}}, \quad b_{74}=\frac{M^{2} \varepsilon^{2} \cos ^{2} \omega t A_{5}}{\left(1+m^{2}\right) q_{m_{1}}\left[m_{5}^{2}+\omega \tan \omega t\right]}, \\
& b_{71}=\frac{-\varepsilon^{3} \cos ^{3} \omega t A_{5} \overline{A_{6}}\left\{m_{5} \overline{m_{6}}\left(1+m^{2}\right)+M^{2}\right\}}{\left(1+m^{2}\right) q_{m_{l}} \overline{q_{m_{1}}}\left[\left(m_{5}+\overline{m_{6}}\right)^{2}+\omega \tan \omega t\right]}, \quad b_{75}=\frac{M^{2} \varepsilon^{2} \cos ^{2} \omega t A_{6}}{\left(1+m^{2}\right) q_{m_{l}}\left[m_{6}^{2}+\omega \tan \omega t\right]}, \\
& b_{72}=\frac{-\varepsilon^{3} \cos ^{3} \omega t A_{6} \overline{A_{5}}\left\{m_{6} \overline{m_{5}}\left(1+m^{2}\right)+M^{2}\right\}}{\left(1+m^{2}\right) q_{m_{l}} \overline{q_{m_{I}}}\left[\left(m_{6}+\overline{m_{5}}\right)^{2}+\omega \tan \omega t\right]}, \quad b_{76}=\frac{M^{2} \varepsilon^{2} \cos ^{2} \omega t \overline{A_{5}}}{\left(1+m^{2}\right)\left[{\overline{m_{5}}}^{2}+\omega \tan \omega t\right]}, \\
& b_{73}=\frac{-\varepsilon^{3} \cos ^{3} \omega t A_{6} \overline{A_{6}}\left\{\overline{m_{6}} m_{6}\left(1+m^{2}\right)+M^{2}\right\}}{\left(1+m^{2}\right) q_{m_{1}} \overline{q_{m_{l}}}\left[\left(m_{6}+\overline{m_{6}}\right)^{2}+\omega \tan \omega t\right]}, \quad b_{77}=\frac{M^{2} \varepsilon^{2} \cos ^{2} \omega t \overline{A_{6}}}{\left(1+m^{2}\right)\left[{\overline{m_{6}}}^{2}+\omega \tan \omega t\right]}, \\
& b_{94}=\frac{-\beta \overline{A_{7}} \overline{m_{7}} \varepsilon \cos \omega t\left\{A_{7} m_{7}\left(1+m^{2}\right)+M^{2} h^{2} \alpha \sigma_{1}\right\}}{\left\{\left(m_{7}+\overline{m_{7}}\right)^{2}+\omega \tan \omega t\right\} \alpha\left(1+m^{2}\right)\left(q_{m_{2}} \overline{q_{m_{2}}}\right)}, \quad b_{100}=\frac{M^{2} h^{2} \overline{A_{7}} \beta \sigma_{1}}{\left(1+m^{2}\right){\overline{q_{m_{2}}}}\left({\overline{m_{7}}}^{2}+\omega \tan \omega t\right)}, \\
& b_{95}=\frac{-\beta A_{7} \overline{A_{8}} \varepsilon \cos \omega t\left\{m_{7} \overline{m_{8}}\left(1+m^{2}\right)+M^{2} h^{2} \alpha \sigma_{1}\right\}}{\left\{\left(m_{7}+\overline{m_{8}}\right)^{2}+\omega \tan \omega t\right\} \alpha\left(1+m^{2}\right)\left(q_{m_{2}} \overline{q_{m_{2}}}\right)}, \quad b_{101}=\frac{M^{2} h^{2} \overline{A_{8}} \beta \sigma_{1}}{\left(1+m^{2}\right) \overline{q_{m_{2}}}\left({\overline{m_{8}}}^{2}+\omega \tan \omega t\right)},
\end{aligned}
$$




$$
\begin{aligned}
& b_{96}=\frac{-\beta A_{8} \varepsilon \cos \omega t\left\{m_{8} \overline{A_{8}} \overline{m_{8}}\left(1+m^{2}\right)+M^{2} h^{2} \overline{A_{7}} \alpha \sigma_{1}\right\}}{\left\{\left(m_{8}+\overline{m_{7}}\right)^{2}+\omega \tan \omega t\right\} \alpha\left(1+m^{2}\right)\left(q_{m_{2}} \overline{q_{m_{2}}}\right)}, \\
& b_{97}=\frac{-\beta A_{8} \varepsilon \cos \omega t\left\{m_{8} \overline{A_{8}} \overline{m_{8}}\left(1+m^{2}\right)+M^{2} h^{2} \alpha \overline{A_{8}} \sigma_{1}\right\}}{\left\{\left(m_{8}+\overline{m_{8}}\right)^{2}+\omega \tan \omega t\right\} \alpha\left(1+m^{2}\right)\left(q_{m_{2}} \overline{q_{m_{2}}}\right)}, \\
& b_{98}=\frac{M^{2} h^{2} A_{7} \beta \sigma_{1} \overline{q_{m_{2}}}}{\left(1+m^{2}\right)\left(q_{m_{2}} \overline{q_{m_{2}}}\right)\left(m_{7}{ }^{2}+\omega \tan \omega t\right)}, \quad b_{99}=\frac{M^{2} h^{2} A_{8} \beta \sigma_{1} \overline{q_{m_{2}}}}{\left(1+m^{2}\right)\left(q_{m_{2}} \overline{q_{m_{2}}}\right)\left(m_{8}{ }^{2}+\omega \tan \omega t\right)} .
\end{aligned}
$$

\section{REFERENCES}

[1] Shail R. (1973): On laminar two-phase flows in magnetohydrodynamics.- Int. J. Engg. Sci., vol.11, pp.1103-1109.

[2] Cowling, T.G. (1962):Magnetohydrodynamics.- Rep. Prog. Phys., vol.25, pp. 244.

[3] Hall Edwin (1879): On a new action of the magnet on electric currents.- American Journal of Mathematics, vol. 2 , No.2, pp.287-292.

[4] BroerL.J.F. and Peletier L.A. (1960): A Mechanical. Hall Effect.- Appl. Sci. Res., vol.8B, pp.259.

[5] Sato H. (1961): The Hall Effect in the viscous flow of ionized gas between parallel plates under transverse magnetic field.- J. Phys. Soc. Japan, vol. 16, No.7, pp.1427-1433.

[6] Tani I. (1962): Steady flow of conducting fluids in channels under transverse magnetic fields with consideration of Hall effect.- J. Aerospace Sci., vol.29, pp.297.

[7] Pop I. (1971): The effect of Hall current on hydromagnetic flow near accelerated plate.- J. Math. Phys. Sci., vol.5, pp.375-379.

[8] CramerKenneth R. and Pai Shih-I. (1973): Magnetofluid Dynamics for Engineers and Applied Physicists.- McGrawHill Company.

[9] Shercliff J.A. (1979): Thermoelectric magnetohydrodynamics.- J.Fluid Mechanics, vol.91, No.2, pp.231-251.

[10] Sharma R.C. and Neela Rani (1988): Hall effects of thermo-solute instability of a plasma.- Indian J. Pure Appl. Math, vol.19, No.2, pp.202-207.

[11] NiranjanS.S., SoundalgekarV.M. and Takhar H.S. (1990): Free convection effects on HMD horizontal channel flow with Hall currents.- Plasma Sci. IEEE Trans, vol.18, No.2, pp.177-183.

[12] Raju T.L and Ramana Rao V.V. (1992): Hall effect in the viscous flow of an ionized gas between two parallel walls under transverse magnetic field in a rotating system.- Acta Physica Hungarica, vol.72, No.1, pp.23-45.

[13] Raju T.L. and Ramana Rao V.V.(1993): Hall effects on temperature distribution in a rotating ionized hydromagnetic flow between parallel walls.- Int. J. Engg. Sci., vol.31, No.7, pp.1073-1091.

[14] Attia H.A. (1998): Hall current effects on the velocity and temperature fields of an unsteady Hartmann flow.- Can. J. Phys, vol.76, No.9, pp.739-746.

[15] AboeldahabE.M. and Elbarbary E.M.E. (2001): Hall current effect on magnetohydrodynamic free convection flow past a semi-infinite vertical plate with mass transfer.- Int. J. Engg. Sci., vol.39, pp.1641-1652.

[16] Singh J.K., Begum S.G. and Seth G.S. (2018): Influence of Hall current and wall conductivity on hydromagnetic mixed convective flow in a rotating Darcian channel.- Physics of fluids, vol.30, No.11, p.12.

[17] Shail R. (1973): On laminar two-phase flows in magnetohydrodynamics.- Int. J. Engg. Sci., vol.11, pp.1103-1109.

[18] ChwlaT.C. and Ishii M. (1980): Two-fluid model of two-phase flow in a pin bundle of anuclear reactor.- Int. J. Heat Mass Transfer, vol.23, pp.991-1001.

[19] Dunn P.F. (1980): Single-phase and two-phase magnetohydrodynamic pipe flow.- Int. Journal of Heat and Mass Transfer, vol.23, pp.373.

[20] Dobran F. (1981): On the consistency conditions of averaging operators in 2-phase flow models and on the formulation of magnetohydrodynamic 2-phase flow.- I. J. Eng. vol.19, No.10, pp.1353-1368. 
[21] Mitra P. (1982): Unsteady flow of two electrically conducting fluids between two rigid parallel plates.- Bulletin of the Calcutta Mathematical Society, vol.74, pp.87-95.

[22] Lohrasbi J. and Sahai V. (1988): Magnetohydrodynamic heat transfer in two phase flow between parallel plates.Appl. Sci. Res., vol.45, pp.53-66.

[23] Setayesh A. and Sahai V. (1990): Heat transfer in developing magnetohydrodynamic Poiseuille flow and variable transport properties.- Int. J. Heat Mass Transfer, vol.33, No.8, pp.1711.

[24] Malashetty M.S. and Leela V. (1992): Magnetohydrodynamic heat transfer in two phase flow.- Int. J. of Engg. Sci., vol.30, pp.371-377.

[25] Chamkha A.J. (200): Unsteady laminar hydromagnetic fluid-particle flow and heat transfer in channels and circular pipes.- Int. J. of Heat and Fluid Flow, vol.21, pp.740-746.

[26] Umavathi J.C., Chamkha A.J., Mateen A. and Kumar J.P. (2008): Unsteady magneto-hydrodynamic two fluid flow and heat transfer in a horizontal channel.- Heat and Technology, vol.26, No.2, pp.121-133.

[27] Tsuyoshi Inoue and Inutsuka Shu-Ichiro (2008): Two-fluid MHD simulations of converging Hi flows in the interstellar medium I:Methodology and basic results.- The Astr. Phys. J., vol.687, pp.303-310.

[28] Hussameddine S. K., Martin J. M. and Sang W. J. (2008): Analytical prediction of flow field in magnetohydrodynamic based micro fluidic devices.- J. of Fluids Engg, vol.130, No.9, pp.6-10.

[29] Srivastava K.M. (2009): Effect of Hall current on the instability of an anisotropic plasma.- Jet. J. Plasma Phys., vol.12, No.1, pp.33-43.

[30] Stamenkovic M.Z., Nikodjevic D.D, Blagojevic B.D. and Savic S. (2010): MHD flow and heat transfer of two immiscible fluids between moving plates.- Transactions of the Canadian Society for Mechanical Engg., vol.34, No.3-4, pp.351-372.

[31] Stamenkovic Ź.M., Nikodijevic D.D., Kocic M.M. and Nikodijevic J.D. (2012): MHD flow and heat transfer of two immiscible fluids with induced magnetic field.- Thermal Science: Int. Scientific Journal, vol.16, No.2, pp.323-336.

[32] Raju T.L and Valli M. (2014): MHD two-layered unsteady fluid flow and heat transferthrough a horizontal channel between parallel plates in a rotating system.- Int. J. Appl. Mech. and Engg., vol.19, No.1, pp.97-121.

[33] Selimli S., Resebli Z. and Arcakhoglu E. (2015): Combined effects of magnetic and electric field on hydrodynamic and thermo-physical parameters of magneto-viscous fluid flow.- Int. J. Heat Mass Trans., vol.86, pp.426-432.

[34] Kalra G.L., Kathuria S.N., Hosking R.J. and Lister G.G. (2009): Effect of Hall current and resistivity on the stability of a gas-liquid system.- J. Plasma Phys., vol.4, No.3, pp.451-469.

[35] Hyun S. and Kennel C.F. (2009): Small amplitude waves in a hot relativistic two-fluid plasma.- J. Plasma Phy., vol.20, No.2, pp.281-287.

[36] Sharma P.R. and Sharma Kalpana (2014): Unsteady MHD two-fluid flow and heat transfer through a horizontal channel.- Int. J. of Engineering Science Invention Research and Development, vol.1, No.3, pp.65-72.

[37] Joseph K.M., Peter A., Asie P.E. and Usman S. (2015): The unsteady MHD free convective two immiscible fluid flows in a horizontal channel with heat and mass transfer.- Int. J. of Mathematics and Computer Research, vol.3, No.5, pp.954-972.

[38] Sivakamini L and Govindarajan A. (2019): Unsteady MHD flow of two immiscible fluids under chemical reaction in a horizontal channel.- AIP conference proceedings $2112.020157 \mathrm{https} / / / \mathrm{doi} .0 \mathrm{rg} / 10.1063 / 1.5112342$, published online: 24 June 2019.

[39] Gireesha B.J., Mahantesh B., Thammanna G.T. and Sampathkumar P.B.(2018): Hall effects on dusty nanofluid twophase transient flow past a stretching sheet using KVL model.- Journal of Molecular Liquids, vol.256, pp.139-147. https://doi.org/10.1016/j.molliq.2018.01.186.

[40] Raju T. L. (2019): MHD heat transfer two-ionized fluids flow between parallel plates with Hall currents.- Journal of Results in Engineering, Elsevier Publication, vol.4, 100043. Available online at https://doi.org/10.1016/j.rineng.2019.100043. 